\title{
Weakly Hyperbolic Cauchy Problem for Second Order Operators
}

By

\author{
Tatsuo NISHITANI*
}

\section{$\S \mathbb{1}$. Introdenction}

We study the $C^{\infty}$ well-posedness of the following Cauchy problem.

$$
\left\{\begin{array}{l}
P\left(t, x, D_{t}, D_{x}\right) u=D_{t}^{2} u-\sum_{j=0}^{2} Q_{j}\left(t, x, D_{x}\right) u+R\left(t, x, D_{x}\right) D_{t} u=f \\
D_{t}^{j} u(0, x)=u_{j}(x), \quad j=0,1
\end{array}\right.
$$

where $Q_{j}\left(t, x, D_{x}\right)$ and $R\left(t, x, D_{x}\right)$ are the differential operators of order $j$ and 0 respectively with $C^{\infty}$-coefficients defined in a neighborhood of the origin in $\mathbb{R}^{d+1}$, and

$$
x=\left(x_{1}, \ldots, x_{d}\right), \xi=\left(\xi_{1}, \ldots, \xi_{d}\right) \in \mathbb{R}^{d}, D_{x}=\left(\frac{1}{i} \frac{\partial}{\partial x_{1}}, \ldots, \frac{1}{i} \frac{\partial}{\partial x_{d}}\right), D_{t}=\frac{1}{i} \frac{\partial}{\partial t}
$$

Denote by $P_{2}(t, x, \tau, \xi)$ and $P^{s}(t, x, \tau, \xi)$ the principal and subprincipal symbols of $\mathbb{P}$. We formulate the assumptions and results in the global version. It is straightforward to give a microlocalized version of the assumptions and results. This is not carried out below.

Modifying $Q_{2}(t, x, \xi)$ near $\xi=0$ and extending it to the outside of a small ball $\{|x|>R\}$, we assume that

$$
\left\{\begin{array}{l}
Q_{2}(t, x, \xi)=\Phi(t, x, \xi)^{2 p} E(t, x, \xi), \text { with real } \Phi(t, x, \xi) \in S_{1,0}^{0}, \\
E(t, x, \xi) \geqq c_{0}\langle\xi\rangle^{2}, \text { with } c_{0}>0,\langle\xi\rangle^{2}=1+\sum_{j=0}^{d} \xi_{j}^{2}, E(t, x, \xi) \in S_{1,0}^{2} .
\end{array}\right.
$$

Communicated by S. Matsuura, March 9, 1983. Revised April 19, 1984.

* Department of Mathematics, Kyoto University, Kyoto 606, Japan.

Present Address : Department of Mathematics, Faculty of General Education, Osaka University, Osaka 560, Japan. 
We also suppose one of the following conditions.

$$
\partial_{t} \Phi(t, x, \xi)=B(t, x, \xi) \bar{\Phi}(t, x, \xi) \text {, with } B(t, x, \xi) \in S_{1,0}^{0} .
$$

This is a global version of the involutive double characteristic case.

$$
\Phi(t, x, \xi)=(t-\phi(x, \xi)) \psi(t, x, \xi),
$$

where $\phi(x, \xi), \phi(t, x, \xi) \in S_{1,0}^{0}$ are real valued and $|\psi(t, x, \xi)| \geqq c>0$. This case corresponds to the non-involutive double characteristic case.

Set $\Lambda_{1}=\tau-\Phi^{p} E^{1 / 2}, \Lambda_{2}=\tau+\Phi^{p} E^{1 / 2}$. Then we have

Theorem 1.1. Assume (1.2) (1.3) or (1.2) (1.4). Then if

$$
\left.\mathbb{P}^{s}\right|_{\tau=0}=C_{1}\left\{\Lambda_{1}, \Lambda_{2}\right\}+C_{2}\left(\Lambda_{1}-\Lambda_{2}\right) \text {, with } C_{i}(t, x, \xi) \in S_{1,0}^{0}
$$

the Cauchy problem (1.1) is $C^{\infty}$ well posed in a neighborhood of the origin. Here $\left\{\Lambda_{1}, \Lambda_{2}\right\}$ denotes the Poisson bracket of $\Lambda_{1}$ and $\Lambda_{2}$.

In the case (1.3), the condition (1.5) is reduced to

$$
\left.P^{s}\right|_{\tau=0}=C(t, x, \xi) \Phi(t, x, \xi)^{p} \text {, with } C(t, x, \xi) \in S_{1,0}^{1} \text {. }
$$

Under this condition (with (1.2) (1.3)), $C^{\infty}$ well-posedness of the Cauchy problem (1.1) was proved in [9], [15].

In the case (1.4), the condition (1.5) is reduced to

$$
\left.P^{s}\right|_{\tau=0}=C(t, x, \xi) \Phi(t, x, \xi)^{p-1} \text {, with } C(t, x, \xi) \in S_{1,0}^{1},
$$

which is equivalent to

$$
Q_{1}(t, x, \xi)=(t-\phi(x, \xi))^{p-1} q(t, x, \xi), \text { with } q(t, x, \xi) \in S_{1,0}^{1} .
$$

If $p=1$, supposing (1.2) (1.4), the $C^{\infty}$ well-posedness of (1.1) follows from [5] (note that $(1.7)^{\prime}$ is satisfied for any $\left.Q_{1}\right)$. When $\phi(x, \xi)=$ const., $p \geqq 1$, the well-posedness of (1.1) was proved in [14], [8], [9], assuming (1.2) (1.4) and (1.7)'. However, we do not know how to reduce the general case to the case when $\phi=$ const., preserving support conditions of solutions.

Therefore, in this paper, we shall prove Theorem 1.1 under the conditions (1.2) (1.4) and (1.7)' when $p \geqq 2$, es`ablishing the energy inequalities, (Theorem 1.2 and 
1.3).

We give some remarks on necessary conditions for the $C^{\infty}$ well posedness of (1.1). Assume that (1.2) and (1.3) are satisfied locally. Then for the $C^{\infty}$ well posedness of (1.1), it is necessary that $\left.\mathbb{P}^{s}\right|_{\tau=0}$ vanishes on $\{\underline{\Phi}=0\}$ locally. Moreover, if $\operatorname{grad}_{t, x} \bar{\Phi}=0$ on $\{\Phi=0\}$, then (1.6) is necessary for the well posedness. ([4]).

If (1.2) and (1.4) are satisfied locally, for the well posedness of (1.1), it is necessary that $(1.7)^{\prime}$ holds locally $([4])$.

Before stating the energy inequalities, we introduce some pseudodifferential operators which will be used throughout this paper. Set

$$
J(t, x, \xi)=\left\{(t-\phi(x, \xi))^{2}+\langle\xi\rangle^{-2 \sigma}\right\}^{1 / 2}, 0<\sigma=1 /(p+1) \leqq 1 / 3 .
$$

We choose $\chi(y) \in C^{\infty}(\mathbb{R})$ so that $\chi(y)=1$ for $y \geqq-1 / 4, \chi(y)=0$ for $y \leqq-1 / 2$ and define

$$
\begin{aligned}
\alpha_{n}^{ \pm}(t, x, \xi)= & \chi\left( \pm n^{1 / 2}(t-\phi(x, \xi))\langle\xi\rangle^{\sigma}\right), \alpha^{ \pm}(t, x, \xi)=\alpha_{1}^{ \pm}(t, x, \xi), \\
J_{ \pm}(t, x, \xi)= & \left(2 \alpha^{ \pm}(t, x, \xi)-1\right)\{ \pm(t-\phi(x, \xi))\}+\langle\xi\rangle^{-\sigma} \\
= & \alpha^{ \pm}(t, x, \xi)\left\{ \pm(t-\phi(x, \xi))+\langle\xi\rangle^{-\sigma}\right\} \\
& +\left(1-\alpha^{ \pm}(t, x, \xi)\right)\left\{\mp(t-\phi(x, \xi))+\langle\xi\rangle^{-\sigma}\right\} .
\end{aligned}
$$

Using these, we introduce the following semi-norm.

$$
\|u\|_{n, s, r}^{2}=\left\|\langle D\rangle^{2 n \sigma} J_{-}(n-r) \alpha_{n}^{-} u\right\|_{s}^{2}+\left\|J_{+}(-n-r) \alpha_{n}^{+} u\right\|_{s^{2}}^{2}
$$

where $J_{ \pm}(k)$ and $\alpha_{n}^{ \pm}$denote the pseudo-differential operator with symbols $J_{ \pm}(t, x, \xi)^{k}$ and $\alpha_{n}^{ \pm}(t, x, \xi)$ respectively and $\|u\|_{s}$ denotes the usual Sobolev norm in $\mathbb{H}^{s}\left(\mathbb{R}^{d}\right)$.

Theorem 1.2. Suppose (1.2), (1.4), (1.7)' and $p \geqq 2$. Then we have

$$
\begin{aligned}
& c(n, N) \int e^{-2 i \theta}\|\mathbb{P} u\|_{-2 N}^{2} d t+c(n, N) \int e^{-2 t \theta}\|\mathbb{P} u\|_{n, 0,0}^{2} d t \\
& \geqq c_{1} n^{1 / 2} \int e^{-2 t \theta}\left\|\mathbb{D}_{t} u\right\|_{n, 0,1}^{2} d t+c_{2} n \int e^{-2 t \theta}\|u\|_{n, 1,1-p}^{2} d t \\
& \quad+c_{3} \theta^{1 / 2} \int e^{-2 t \theta}\left\|D_{t} u\right\|_{n, 0,1 / 2}^{2} d t+c_{3} \theta^{1 / 2} \int e^{-2 t \theta}\|u\|_{n, 1,1 / 2-p}^{2} d t \\
& \quad+c_{4} \theta^{1+1 / 4} \int e^{-2 t \theta}\|u\|_{-N}^{2} d t+c_{4} \theta^{2+3 / 4} \int e^{-2 i \theta}\left\|D_{t} u\right\|_{-N}^{2} d t
\end{aligned}
$$

for $n \geqq c\left(Q_{2}\right) C, \quad \theta \geqq \theta_{0}(n, N), N \geqq 1, \quad u \in C_{0}^{\infty}\left((-T, T) \times \mathbb{R}^{d}\right)$, where $C=$ $\sup \left|J(t, x, \xi)^{-p+1}\langle\xi\rangle^{-1} Q_{1}(t, x, \xi)\right|, c_{i}>0(1 \leqq i \leqq 3), c_{4}=c_{4}(n, N)>0$, and 
$c\left(Q_{2}\right)$ depends only on $Q_{2}(t, x, \xi)$.

Theorem 1.3. Assume the same hypothesis as above. Then the following inequality holds,

$$
c(n, s) \int e^{-2 t \theta}\|P u\|_{2 n \sigma+s+1}^{2} d t \geqq \theta^{1+1 / 4} \int e^{-2 t \theta}\left\|D_{t} u\right\|_{s}^{2} d t+\theta^{2+3 / 4} \int e^{-2 t \theta}\|u\|_{s}^{2} d t,
$$

for $\theta \geqq \theta_{0}(n, s), n \geqq c\left(Q_{2}\right) C, s \in \mathbb{R}, u \in C_{0}^{\infty}\left((-T, T) \times \mathbb{R}^{d}\right)$.

Remark 1。且。 We note that

$$
\langle\xi\rangle^{2 n \sigma} J_{-}(t, x, \xi)^{n} \alpha_{n}^{-}(t, x, \xi)+J_{+}(t, x, \xi)^{-n} \alpha_{n}^{+}(t, x, \xi)
$$

is equivalent to 1 when $t-\phi(x, \xi) \geqq c$, to $\langle\xi\rangle^{n \sigma}$ when $|t-\phi(x, \xi)| \leqq c\langle\xi\rangle^{-\sigma}$ to $\langle\xi\rangle^{2 n \sigma}$ when $t-\phi(x, \xi) \leqq-c$ with arbitrary positive $c$. Then, since this Cauchy problem (1.1) may cause a great loss of differentiability of solution, the use of this semi-norm \|\|$u \|_{n, s, r}$ seems to be natural.

\section{§ 2. Some Properties on Symbols in $\mathbb{J}^{r} \mathbb{S}^{m}$}

We shall say that $a(t, x, \xi) \in C^{\infty}\left(I \times \mathbb{R}^{2 d}\right), I=(-T, T)$ belongs to $S_{\rho, \delta}^{m}$ (Hörmander's class) if

$$
\left|\partial_{t}^{j} a_{(\beta)}^{(\alpha)}(t, x, \xi)\right| \leqq C_{j, \alpha, \beta}\langle\xi\rangle^{m-\beta|\alpha|+\delta(|\beta|+j)},
$$

for any multi-index $\alpha, \beta \in \mathbb{N}^{d}$. We denote by $J^{r} S^{m}$ the set of all $a(t, x, \xi) \in$ $C^{\infty}\left(I \times \mathbb{R}^{2 d}\right)$ such that

$$
\left|\partial_{t}^{j} a_{(\beta)}^{(\alpha)}(t, x, \xi)\right| \leqq C_{j, \alpha, \beta} J(t, x, \xi)^{r-j-|\alpha+\beta|}\langle\xi\rangle^{m-|\alpha|},
$$

for all multi-indexes $\alpha, \beta \in \mathbb{N}^{d}$. This class is a variant one treated in [2], also see [1]. But in this paper we always treat the variable $t$ as a parameter.

The pseudodifferential operator with symbol $a(t, x, \xi)$ belonging to one of the above classes is realized by

$$
a\left(t, x, D_{x}\right) u=\int e^{i x \xi} a(t, x, \xi) \widehat{u}(\xi) d \xi, \quad \widehat{u}(\xi)=\int e^{-i x \xi} u(x) d x
$$

For the pseudodifferential operator $a\left(t, x, D_{x}\right)$, we denote by $\sigma(a)(t, x, \xi)$ the symbol of $a\left(t, x, D_{x}\right)$. But sometimes we do not distinguish the operator and its symbol. 
Proposition 2。H。 $\langle\xi\rangle^{-\sigma},(t-\phi(x, \xi)) \in J S^{0}, \alpha_{n}^{ \pm}(t, x, \xi) \in J^{0} S^{0}, S_{1,0}^{m} \subset$ $J^{0} S^{m}, J^{r} S^{m} \subset S_{1-\sigma, \sigma}^{m+\sigma r-}$ where $r^{-}=\max (0,-r)$.

Proposition 2.2. $c_{1} J(t, x, \xi) \geqq J_{ \pm}(t, x, \xi) \geqq c_{2} J(t, x, \xi)$ with $\quad c_{i}>0 . \quad \mathbb{I} f$ $a(t, x, \xi) \in J^{r} S^{m}$ then $\partial_{t}^{j} a_{\beta}^{(\alpha)}(t, x, \xi) \in J^{r-j-|\alpha+\beta|} S^{m-|\alpha|}$.

Proof. We shall show the first inequalities for $J_{+}(t, x, \xi)$. When $(t-\phi(x$, $\xi)\langle\xi\rangle^{\sigma} \geqq 0$ or $(t-\phi(x, \xi))\langle\xi\rangle^{\sigma} \leqq-1 / 2$, we have $J_{+}(t, x, \xi)=|t-\phi(x, \xi)|$ $+\langle\xi\rangle^{-\sigma}$ from the definition, and the inequalities are immediate. When $-1 / 2 \leqq$ $(t-\phi(x, \xi))\langle\xi\rangle^{\sigma} \leqq 0$, it follows that

$$
J_{+}(t, x, \xi) \geqq\langle\xi\rangle^{-\sigma}-|t-\phi(x, \xi)| \geqq 4^{-1}\left\{|t-\phi(x, \xi)|+\langle\xi\rangle^{-\sigma}\right\} .
$$

Then we obtain the desired inequalities.

Proposition 2.3. If $a_{i}(t, x, \xi) \in J^{r_{i}} S^{m_{i}}(i=1,2)$, then $a_{1}(t, x, \xi) a_{2}(t, x, \xi)$ $\in J^{r_{1}+r_{2}} S^{m_{1}+m_{2}}$. If $a(t, x, \xi) \in J^{r} S^{m}$ and $a(t, x, \xi) \geqq c\langle\xi\rangle^{m} J(t, x, \xi)^{r}$ with $c>$ 0 , then $a(t, x, \xi)^{n} \in J^{r n} S^{m n}$, for $n \in \mathbb{R}$.

Corollary 2.1. $J(t, x, \xi)^{n}, J_{ \pm}(t, x, \xi)^{n} \in J^{n} S^{0}$, for $n \in \mathbb{R}$.

Proposition 2.4. Let $a_{i}\left(i, x, D_{x}\right) \in J^{r_{i}} S^{m_{i}}(i=1,2)$, then $a_{1}\left(t, x, D_{x}\right) a_{2}(t$, $\left.x, D_{x}\right) \in J^{r_{1}+r_{2}} S^{m_{1}+m_{2}}$. If $a\left(t, x, D_{x}\right) \in J^{r} S^{m}$, then $a\left(t, x, D_{x}\right) *$ belongs io $J^{r} S^{m}$, where $a\left(t, x, D_{x}\right) *$ denotes the adjoint of $a\left(t, x, D_{x}\right)$ with respect to the scalar product in $L^{2}\left(\mathbb{R}^{d}\right)$.

Proof. (See [2], [1].) For the proof, we introduce the following weight function

$$
d(y, \xi, \eta)=1+\langle\xi\rangle|y|^{2}+\langle\xi\rangle^{-1}|\eta|^{2}
$$

First we note the following inequalities.

$$
\begin{gathered}
J(t, x+y, \xi)^{s} \leqq C_{s} J(t, x, \xi)^{s} d(y, \xi, \eta)^{|s| / 2} \\
J(t, x, \xi+\theta \eta)^{s} \leqq C_{s} J(t, x, \xi)^{s} d(y, \xi, \eta)^{|s| / 2}, 0 \leqq \theta \leqq 1, \text { for }|\xi| \geqq 2(1+|\eta|), \\
J(t, x, \xi+\theta \eta)^{s} \leqq C_{s} J(t, x, \xi)^{s}\langle\eta\rangle^{(1+\sigma)|s|}, 0 \leqq \theta \leqq 1, \text { for }\left|\xi^{\xi}\right| \leqq 2(1+|\eta|), \\
\left|\alpha_{\xi}^{\gamma}\left(\langle\xi\rangle \Delta_{\eta}\right)^{i}\left(\langle\xi\rangle^{-1} \Delta_{y}\right)^{j} d(y, \xi, \eta)^{-K}\right| \leqq C_{K, i, j, \gamma}\langle\xi\rangle^{-|\gamma|} d(y, \xi, \eta)^{-K}
\end{gathered}
$$

Now we set $C_{\theta}(t, x, y, \xi, \eta)=a_{1}(t, x, \xi+6 \eta) a_{2}(t, x+y, \xi)$ and consider the 
following oscillatory integral

$$
\mathbb{I}(C)=\iint e^{-i y \eta} C_{\theta}(t, x, y, \xi, \eta) d y d \eta=\mathbb{I}_{1}\left(C_{\theta}\right)+\mathbb{I}_{2}\left(C_{\theta}\right)
$$

where $I_{1}\left(C_{\theta}\right)$ and $I_{2}\left(C_{\theta}\right)$ denote the integrals on $|\xi| \geqq 2(1+|\eta|)$ and $|\xi| \leqq 2(1+$ $|\eta|)$ respectively. First we treat $\mathbb{I}_{1}\left(C_{\theta}\right)$,

$$
\begin{gathered}
\partial_{x}^{\alpha} \partial_{\xi}^{\gamma} I_{1}\left(C_{\theta}\right)=\iint e^{-i y \eta} \partial_{x}^{\alpha} \partial_{\xi}^{\gamma}\left(1-\langle\xi\rangle \Delta_{\eta}-\langle\xi\rangle^{-1} \Delta_{y}\right)^{K} \\
\times\left(d(y, \xi, \eta)^{-K} C_{\theta}(t, x, y, \xi, \eta)\right) d y d \eta .
\end{gathered}
$$

Using the estimates (2.3), (2.4) and (2.6), the integrand is estimated by

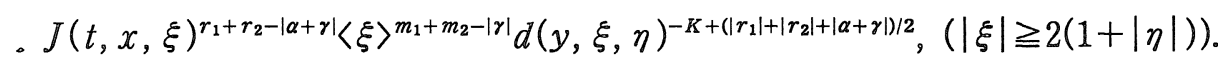

Since

$$
\iint\left(1+\langle\xi\rangle|y|^{2}+\langle\xi\rangle^{-1}|\eta|^{2}\right)^{-K+\left(\left|r_{1}\right|+\left|r_{2}\right|+|\alpha+\gamma|\right) / 2} d y d \eta \leqq C
$$

with suitable $K=K\left(r_{1}, r_{2}, \alpha, \gamma\right)$, we have the desired estimates (that is, in $\left.J^{r_{1}+r_{2}} S^{m_{1}+m_{2}}\right)$ for $\mathbb{I}_{1}\left(C_{\theta}\right)$.

We proceed to the next,

$$
\partial_{x}^{\alpha} \partial_{\xi}^{\gamma} I_{2}\left(C_{\theta}\right)=\iint e^{-i y \eta} \partial_{x}^{\alpha} \partial_{\xi}^{\gamma}\langle\eta\rangle^{-2 N}\left(1-\Delta_{y}\right)^{N}\langle y\rangle^{-2 M}\left(1-\Delta_{\eta}\right)^{M} C_{\theta}(t, x, y, \xi, \eta) d y d \eta
$$

Remarking the inequalities (2.3) and (2.5), the above integrand is estimated by

$$
J(t, x, \xi)^{r_{1}+r_{2}-|\alpha+\gamma|}\langle\xi\rangle^{m_{1}+m_{2}-|\gamma|}\langle y\rangle^{-d-1}\langle\eta\rangle^{-d-1}
$$

Here we have taken $N, M$ so that $-2 M+\left|r_{2}\right|+|\alpha+\gamma| \leqq-d-1,-N+(1+\sigma)$ $\times\left(\left|r_{1}\right|+\left|r_{2}\right|+|\alpha+\gamma|\right)+\left|m_{1}\right|+|\gamma| \leqq-d-1$. Therefore it follows that

$$
\left|\partial_{x}^{\alpha} \partial_{\xi}^{\gamma} I\left(C_{\theta}\right)\right| \leqq C_{\alpha, \gamma} J(t, x, \xi)^{r_{1}+r_{2}-|\alpha+\gamma|}\langle\xi\rangle^{m_{1}+m_{2}-|\gamma|}, 0 \leqq \theta \leqq 1
$$

Hence, taking $\theta=0$, we have the first assertion. The second assertion will be proved by the similar arguments.

This proof also shows that

Proposition 2.5. Let $a_{i}\left(t, x, D_{x}\right) \in J^{r} S^{m_{i}}(i=1,2)$, then $\sigma\left(a_{1}(t, x\right.$, 
$\left.\left.D_{x}\right) a_{2}\left(t, x, D_{x}\right)\right)-\sum_{|\alpha| \leq N-1}(\alpha !)^{-1} a_{1}^{(\alpha)}(t, x, \xi) a_{2(\alpha)}(t, x, \xi) \in J^{r_{1}+r_{2}-2 N} S^{m_{1}+m_{2}-N}$ If $a\left(t, x, D_{x}\right) \in J^{r} S^{m}$, then $\sigma\left(a\left(t, x, D_{x}\right)^{* *}\right)-\sum_{|\alpha| \leq N-1}(\alpha !)^{-1}(-1)^{|\alpha|} \overline{\alpha_{\alpha}^{(\alpha)}(t, x, \xi)} \in$ $J^{r-2 N} S^{m-N}$.

The standard method of constructing a symbol from its asymptotic expansion gives that

Proposition 2.6. Let $a_{j}(t, x, \xi) \in J^{r-j} S^{m-j / 2}, j=0,1,2, \cdots$. Then there is $a$ symbol $a(t, x, \xi) \in J^{r} S^{m}$ such that

$$
a(t, x, \xi)-\sum_{j=0}^{N-1} a_{j}(t, x, \xi) \in J^{r-N} S^{m-N / 2} \text { for } N=0,1,2, \cdots
$$

If $\operatorname{supp}\left[a_{j}(t, x, \xi)\right] \subset \mathbb{E}, j=0,1,2, \cdots$, we can take $a(t, x, \xi)$ so that $\operatorname{supp}[a(t, x, \xi)] \subset \mathbb{E}$.

Proposition 2.\%. Suppose that $A_{i}(t, x, \xi) \in J^{r_{i}} S^{m_{i}}(i=1,2), B \in J^{r} S^{m}$ and $A_{i}(t, x, \xi) \geqq c_{i}\langle\xi\rangle^{m_{i}} J(t, x, \xi)^{r_{i}}$, on $\operatorname{supp}[B(t, x, \xi)]$, with $c_{i}>0$ for large $|\xi|$. Then there exists $C(t, x, \xi) \in J^{r-\left(r_{1}+r_{2}\right)} S^{m-\left(m_{1}+m_{2}\right)}$ such that $A_{1} C A_{2}=B, \bmod S^{-\infty}$ and $\operatorname{supp}[C(t, x, \xi)] \subset \operatorname{supp}[B(t, x, \xi)]$.

Lemma 2.1. Let $J_{1} \in J^{k} S^{0}, J_{2} \in J^{k+1 / 2} S^{0}$ and $J_{2}(t, x, \xi) \geqq c_{2} J(t, x, \xi)^{k+1 / 2}$, with $c_{2}>0$ for large $|\xi|$. Then if $A(t, x, \xi) \in J^{0} S^{0}$ and $|A(t, x, \xi)| \leqq \hat{c}$, we have

$$
\left\|A J_{1} u\right\|^{2} \leqq \hat{c}\left\|J_{1} u\right\|^{2}+C(N)\left\|J_{2} u\right\|^{2}+C(N)\|u\|_{-N}^{2} \text { for any } N \text {. }
$$

Proof. First it is clear from Proposition 2.5 that

$$
A^{*} A=o p\left(|A(t, x, \xi)|^{2}\right)-B_{1}, B_{1} \in J^{-2} S^{-1}
$$

The proof of the sharp Garding inequality (see [7]) shows that

$$
\begin{aligned}
& \sigma\left(\mathrm{op}\left(\hat{c}^{2}-|A(t, x, \xi)|^{2}\right)\right) \sim\left(\hat{c}^{2}-|A(t, x, \xi)|^{2}\right)_{F} \\
& \quad-\sum_{|\beta|=1} \psi_{\beta}\left(|A(t, x, \xi)|^{2}\right)_{(\beta)}-\sum_{|\alpha+\beta| \geq 2} \psi_{\alpha, \beta}\left(|A(t, x, \xi)|^{2}\right)(\alpha)
\end{aligned}
$$

where $\psi_{\beta} \in S_{1,0}^{-1}, \psi_{\alpha, \beta} \in S_{1,0}^{(|\alpha|-|\beta|) / 2}$ and $A(t, x, \xi)_{F}$ denotes the Friedrichs symmetrization of $A(t, x, \xi)$. Remarking

$$
\psi_{\beta}\left(|A(t, x, \xi)|^{2}\right)_{(\beta)} \in J^{-1} S^{-1}, \psi_{\alpha, \beta}\left(|A(t, x, \xi)|^{2}\right)_{(\alpha)}^{(\alpha)} \in J^{-2-(|\alpha+\beta|-2)} S^{-(|\alpha+\beta|-2) / 2-1},
$$


and applying Proposition 2.6, we can write

$$
\operatorname{op}\left(\widehat{c}^{2}-|A(t, x, \xi)|^{2}\right)=\left(\widehat{c}^{2}-|A(t, x, \xi)|^{2}\right)_{F}+B_{2}, \bmod S^{-\infty} \text {, with } B_{2} \in J^{-2} S^{-1} \text {. }
$$

This gives that

$$
\widehat{c}^{2}-A^{*} A=\left(\widehat{c}^{2}-|A(t, x, \xi)|^{2}\right)_{F}+B_{3}, \bmod S^{-\infty}, \text { with } B_{3} \in J^{-2} S^{-1},
$$

and hence

$$
\widehat{c}^{2}\|v\|^{2}+\left|\left(B_{3} v, v\right)\right|+C(N)\|v\|_{-N-\sigma|k|}^{2} \geqq\|A v\|^{2} .
$$

Whereas, from Proposition 2.7, one can write

$$
J_{1}^{*} B_{3} J_{1}=J_{2}^{*} B_{4} J_{2}, \bmod S^{-\infty}, \text { with } B_{4} \in J^{-3} S^{-1} \text {. }
$$

Here we note that

$$
-1+3 \sigma \leqq 0
$$

and this implies that $B_{4} \in J^{0} S^{0} \subset S_{1-\sigma, \sigma}^{0}$. Taking into account that (2.8) and $J_{1} \in$ $J^{k} S^{0} \subset S_{1-\sigma, \sigma}^{\sigma|k|}$, we get this lemma by replacing $v$ by $J_{1} u$ in (2.7).

Next let us consider

$$
Q_{M}\left(t, x, D_{x}\right)=Q_{2}\left(t, x, D_{x}\right)+M\left(J(p-1)^{*}\langle D\rangle J(p-1)+\langle D\rangle^{-2 L}\right),
$$

where $Q_{2}(t, x, \xi)$ is the symbol mentioned in Section 1 .

Lemma 2.2. Suppose that $c_{0}>0$ is given. Then there exists positive $c\left(Q_{2}, c_{0}\right)$ such that for any positive integer $L$, we have

$$
\operatorname{Re}\left(Q_{M} u, u\right)+c_{0}\left\|J_{ \pm}(-1) u\right\|^{2} \geqq c\left(Q_{2}, c_{0}\right)\left\|\langle D\rangle J_{ \pm}(p) u\right\|^{2},
$$

with some positive $M$.

Proof. Let us set

$$
L_{ \pm}(t, x, \xi)=J_{ \pm}(t, x, \xi)^{-2 p}\left(Q_{2}(t, x, \xi)+c_{0} J_{ \pm}^{-2}(t, x, \xi)\right),
$$

and first show that

$$
L_{ \pm}(t, x, \xi) \in J^{0} S^{2}
$$


Propositions 2.1 and 2.3 imply that $Q_{2}(t, x, \xi) \in J^{2 p} S^{2}$ and hence $J_{ \pm}(t, x, \xi)^{-2 p} Q_{2}(t, x, \xi) \in J^{0} S^{2}$. The other hand, the equality

$$
2(p+1) \sigma=2
$$

shows $J_{ \pm}(t, x, \xi)^{-2 p-2} \in J^{-2 p-2} S^{0} \subset J^{0} S^{2}$, then we get (2.11).

Next we observe $Q_{2}+c_{0} J_{ \pm}(-1) * J_{ \pm}(-1)-J_{ \pm}(p) * L_{ \pm} J_{ \pm}(p)$. Taking (2.11) into account, Proposition 2.7 shows that

$$
\begin{gathered}
Q_{2}+c_{0} J_{ \pm}(-1) * J_{ \pm}(-1)-J_{ \pm}(p) * L_{ \pm} J_{ \pm}(p) \\
=J(p-1) * M_{ \pm} J(p-1), \bmod S^{-\infty}
\end{gathered}
$$

with $M_{ \pm} \in J^{0} S^{1}$, here we have used the fact $J^{-4} S^{-1} \subset J^{2 p-2} S^{1}$ (follows from (2.12)). Therefore to prove this lemma, it suffices to consider $L_{ \pm}$. Since

$$
\begin{aligned}
L_{ \pm}(t, x, \xi) & =J_{ \pm}(t, x, \xi)^{-2 p-2}\langle\xi\rangle^{2}\left(Q_{2}(t, x, \xi)\langle\xi\rangle^{-2} J_{ \pm}(t, x, \xi)^{2}+c_{0}\langle\xi\rangle^{-2}\right) \\
& \geqq c_{1}\langle\xi\rangle^{2} J_{ \pm}(t, x, \xi)^{-2 p-2}\left((t-\phi(x, \xi))^{2 p+2}+\langle\xi\rangle^{-2}\right), c_{1}>0,
\end{aligned}
$$

in view of the identity $\langle\xi\rangle^{-2}=\langle\xi\rangle^{-2(p+1) \sigma}$, we have

$$
L_{ \pm}(t, x, \xi) \geqq c\left(Q_{2}, c_{0}\right)\langle\xi\rangle^{2} \text {, with } c\left(Q_{2}, c_{0}\right)>0 \text {. }
$$

Let us put

$$
Q_{ \pm}(t, x, \xi)=L_{ \pm}(t, x, \xi)-c\left(Q_{2}, c_{0}\right)\langle\xi\rangle^{2}\left(\in J^{0} S^{2}\right)
$$

and $\psi_{\beta}, \phi_{\alpha, \beta}$ be the same as in the proof of Lemma 2.1. Then we see that

$$
\psi_{\beta} Q_{ \pm(\beta)} \in J^{-1} S^{1}, \psi_{\alpha, \beta} Q_{ \pm}\left(\begin{array}{l}
\alpha \\
\beta
\end{array}\right) \in J^{-2-(|\alpha+\beta|-2)} S^{1-(|\alpha+\beta|-2) / 2}
$$

From the same reasoning in Lemma 2.1, one has

$$
Q_{ \pm}(t, x, \xi)_{F}=Q_{ \pm}(t, x, \xi)+K_{1}^{ \pm}, \bmod S^{-\infty} \text {, with } K_{1}^{ \pm} \in J^{-2} S^{1}
$$

and then it follows that

$$
\operatorname{Re}\left(L_{ \pm} v, v\right)+C(L)\|v\|_{-L}^{2}+\left|\left(K_{1}^{ \pm} v, v\right)\right| \geqq c\left(Q_{2}, c_{0}\right)\|\langle D\rangle v\|^{2}
$$

Take $v=J_{ \pm}(p) u$ in (2.15) and remark that

$$
J_{ \pm}(p)^{*} K_{1}^{ \pm} J_{ \pm}(p)=J(p-1)^{*} K_{2}^{ \pm} J(p-1), \bmod S^{-\infty}, \text { with } K_{2}^{ \pm} \in J^{0} S^{1},
$$

then it follows immediately that 


$$
\begin{aligned}
& C(L)\|u\|_{-L}^{2}+\operatorname{Re}\left(J_{ \pm}(p)^{*} L_{ \pm} J_{ \pm}(p) u, u\right)+C(L)\left\|\langle D\rangle^{1 / 2} J(p-1) u\right\|^{2} \\
& \quad \geqq c\left(Q_{2}, c_{0}\right)\left\|\langle D\rangle J_{ \pm}(p) u\right\|^{2} .
\end{aligned}
$$

Thus (2.13) and (2.16) prove this Lemma.

\section{§ 3. Energy Imequalinty}

Set $\quad I_{n}(m)=\langle D\rangle^{2 n \sigma} J_{-}(m), \quad E_{c}^{ \pm}=\left\{(t, x, \xi) ; \mp(t-\phi(x, \xi)) \leqq c\langle\xi\rangle^{-\sigma}\right\}$, $E_{c}=E_{c}^{+} \cap E_{c}^{-}$. We start with the following identity,

$$
\begin{aligned}
& -2 \operatorname{Im} \int\left(I_{n}(n-1 / 2)\left(D_{t}-i \theta\right)^{2} w, I_{n}(n-1 / 2)\left(D_{t}-i \theta\right) w\right) d t \\
& =2 \theta \int\left\|I_{n}(n-1 / 2)\left(D_{t}-i \theta\right) w\right\|^{2} d t \\
& \quad-2 \operatorname{Re} \int\left(\partial_{t} I_{n}(n-1 / 2)\left(D_{t}-i \theta\right) w, I_{n}(n-1 / 2)\left(D_{t}-i \theta\right) w\right) d t,
\end{aligned}
$$

where $w=\alpha_{n}^{-} u$.

Since $\alpha^{-}(t, x, \xi)=1$ on $E_{1 / 4}^{-}$, we have

$$
\partial_{t} J_{-}(t, x, \xi)^{n-1 / 2}+(n-1 / 2) J_{-}(t, x, \xi)^{n-3 / 2}=0 \text {, on } \operatorname{supp}\left[\alpha_{n}^{-}\right] \subset E_{1 / 2 \sqrt{n}}^{-} .
$$

This means that $\partial_{t} I_{n}(n-1 / 2) \alpha_{n}^{-}=-(n-1 / 2) I_{n}(n-3 / 2) \alpha_{n}^{-}$, $\bmod S^{-\infty}$, and then it follows that

$$
\begin{aligned}
& \int\left(\partial_{t} I_{n}(n-1 / 2)\left(D_{t}-i \theta\right) w, I_{n}(n-1 / 2)\left(D_{t}-i \theta\right) w\right) d t \\
& \quad=-(n-1 / 2) \int\left(I_{n}(n-3 / 2)\left(D_{t}-i \theta\right) w, I_{n}(n-1 / 2)\left(D_{t}-i \theta\right) w\right) d t, \\
& \quad \bmod c(n)\left\{\int\left(\left\|\left(D_{t}-i \theta\right) u\right\|_{-N}^{2}+\|u\|_{-N}^{2}\right) d t\right\}=c(n)[u]_{-N}^{2},(n \geqq 4) .
\end{aligned}
$$

Here we remark that the positive integer $N$ can be taken arbitrarily large, and everywhere below we fix such one $N$.

Using this, the second term of the right-hand side of (3.1) is equivalent to

$$
(2 n-1) \operatorname{Re} \int\left(I_{n}(n-3 / 2)\left(D_{t}-i \theta\right) w, \quad I_{n}(n-1 / 2)\left(D_{t}-i \theta\right) w\right) d t,
$$

$\bmod c(n)[u]_{-N}^{2}$ 
On the other hand, from Proposition 2.7, one can find $B \in J^{-3} S^{-1}$ so that

$$
\begin{gathered}
\mathbb{I}_{n}(n-1 / 2) * \mathbb{I}_{n}(n-3 / 2)=\mathbb{I}_{n}(n-1) * \mathbb{I}_{n}(n-1)+\mathbb{I}_{n}(n-1 / 2) * \mathbb{B} \mathbb{I}_{n}(n-1 / 2), \\
\bmod S^{-\infty}
\end{gathered}
$$

In virtue of (2.9), $B$ belongs to $S_{1-\sigma, \sigma}^{0}$ and then (3.3) is estimated from below by

$$
\begin{aligned}
& (2 n-1) \int\left\|I_{n}(n-1)\left(D_{t}-i \theta\right) w\right\|^{2} d t \\
& \quad-c(n) \int\left\|I_{n}(n-1 / 2)\left(D_{t}-i \theta\right) w\right\|^{2} d t, \bmod c(n)[u]_{-N}^{2}
\end{aligned}
$$

Combining these inequalities, we get for $\theta \geqq \theta_{0}(n)$,

$$
\begin{gathered}
-2 \operatorname{Im} \int\left(\mathbb{I}_{n}(n-1 / 2)\left(\mathbb{D}_{t}-i \theta\right)^{2} w, \mathbb{I}_{n}(n-1 / 2)\left(\mathbb{D}_{t}-i \theta\right) w\right) d t \\
\geqq c_{0} \theta \int\left\|\mathbb{I}_{n}(n-1 / 2)\left(\mathbb{D}_{t}-i \theta\right) w\right\|^{2} d t \\
+(2 n-1) \int\left\|\mathbb{I}_{n}(n-1)\left(\mathbb{D}_{t}-i \theta\right) w\right\|^{2} d t \\
\bmod c(n)[u]_{-N}^{2} .
\end{gathered}
$$

Next consider

$$
\begin{aligned}
-2 \operatorname{Im} & \int\left(J_{-}(-1) \mathbb{I}_{n}(n-1 / 2)\left(\mathbb{D}_{t}-i \theta\right) w, J_{-}(-1) \mathbb{I}_{n}(n-1 / 2) w\right) d t \\
& =2 \theta \int\left\|J_{-}(-1) \mathbb{I}_{n}(n-1 / 2) w\right\|^{2} d t \\
& -2 \operatorname{Re} \int\left(\partial_{t} J_{-}(-1) \mathbb{I}_{n}(n-1 / 2) w, J_{-}(-1) \mathbb{I}_{n}(n-1 / 2) w\right) d t \\
& -2 \operatorname{Re} \int\left(J_{-}(-1) \partial_{t} \mathbb{I}_{n}(n-1 / 2) w, J_{-}(-1) \mathbb{I}_{n}(n-1 / 2) w\right) d t .
\end{aligned}
$$

From the same reasoning as above, we can replace $\partial_{t} J_{-}(-1), \partial_{t} J_{-}(n-1 / 2)$ by $-J_{-}(-2),-(n-1 / 2) J_{-}(n-3 / 2)$ in (3.5) within modulo $c(n)[u]_{-N}^{2}$. Therefore, applying Proposition 2.7, the same arguments imply that the right-hand side of (3.5) is estimated from below by

$$
\begin{aligned}
& c_{0} \theta \int\left\|J_{-}(-1) \mathbb{I}_{n}(n-1 / 2) w\right\|^{2} d t \\
& \quad+(2 n-3) \int\left\|\mathcal{J}_{-}(-1) \mathbb{I}_{n}(n-1) w\right\|^{2} d t, \bmod [u]_{-N}^{2},
\end{aligned}
$$

for $\theta \geqq \theta_{0}(n)$. Now we consider the left-hand side of (3.5). From Proposition 
2.7, we get

$$
\begin{aligned}
& I_{n}(n-1 / 2)^{*} J_{-}(-1)^{*} J_{-}(-1) I_{n}(n-1 / 2)=I_{n}(n-1)^{*} J_{-}(-1)^{*} I_{n}(n-1) \\
& \quad+I_{n}(n-1 / 2)^{*} J_{-}(-1)^{*} B I_{n}(n-1 / 2), \text { with } B \in J^{-3} S^{-1}, \bmod S^{-\infty},
\end{aligned}
$$

and then the left side of (3.5) is estimated by

$$
\begin{gathered}
\int\left\|I_{n}(n-1)\left(D_{t}-i \theta\right) w\right\|^{2} d t+\int\left\|J_{-}(-1) I_{n}(n-1) w\right\|^{2} d t \\
+c(n) \theta^{-1 / 2} \int\left\|I_{n}(n-1 / 2)\left(D_{t}-i \theta\right) w\right\|^{2} d t \\
+c(n) \theta^{1 / 2} \int\left\|J_{-}(-1) I_{n}(n-1 / 2) w\right\|^{2} d t \\
\bmod c(n)\left\{\theta^{1 / 2} \int\|u\|_{-N}^{2} d t+\theta^{-1 / 2} \int\left\|\left(D_{t}-i \theta\right) w\right\|_{-N}^{2} d t\right\}=c(n)[u]_{-N, \theta}^{2}
\end{gathered}
$$

Therefore it follows from (3.6) that

$$
\begin{aligned}
& \int\left\|I_{n}(n-1)\left(D_{t}-i \theta\right) w\right\|^{2} d t \geqq c_{0} \theta \int\left\|J_{-}(-1) I_{n}(n-1 / 2) w\right\|^{2} d t \\
& \quad+(2 n-4) \int\left\|J_{-}(-1) I_{n}(n-1) w\right\|^{2} d t \\
& \quad-c(n) \theta^{-1 / 2} \int\left\|I_{n}(n-1 / 2)\left(D_{t}-i \theta\right) w\right\|^{2} d t
\end{aligned}
$$

for $\theta \geqq \theta_{0}(n), \bmod c(n)[u]_{-N, \theta}^{2}$.

Combining (3.4) with (3.7), we get,

\section{Proposition 3.1.}

$$
\begin{aligned}
& -2 \operatorname{Im} \int\left(I_{n}(n-1 / 2)\left(D_{t}-i \theta\right)^{2} w, I_{n}(n-1 / 2)\left(D_{t}-i \theta\right) w\right) d t \\
& \quad \geqq c_{1} \theta \int\left\|I_{n}(n-1 / 2)\left(D_{t}-i \theta\right) w\right\|^{2} d t+n \int\left\|I_{n}(n-1)\left(D_{t}-i \theta\right) w\right\|^{2} d t \\
& \quad+c_{1} \theta^{2} \int\left\|J_{-}(-1) I_{n}(n) w\right\|^{2} d t+c_{1} n \theta \int\left\|J_{-}(-1) I_{n}(n-1 / 2) w\right\|^{2} d t \\
& \quad+2^{-1} n^{2} \int\left\|J_{-}(-1) I_{n}(n-1) w\right\|^{2} d t, \bmod c(n) \theta[u]_{-N, \theta}^{2}, \text { for } \theta \geqq \theta_{0}(n), n \geqq 4
\end{aligned}
$$

\section{§ 4. Emergy Inequality (Continued)}

We proceed to the next step of obtaining the energy inequality. Consider the identity, 
(4.1) $2 \operatorname{Im} \int\left(I_{n}(n-1 / 2) Q_{M} w, I_{n}(n-1 / 2)\left(D_{t}-i \theta\right) w\right) d t$

$=2 \theta \operatorname{Re} \int\left(Q_{M} I_{n}(n-1 / 2) w, I_{n}(n-1 / 2) w\right) d t$

$-\int\left\{\left(Q_{M} \partial_{t} I_{n}(n-1 / 2) w, I_{n}(n-1 / 2) w\right)+\left(Q_{M} I_{n}(n-1 / 2) w, \partial_{t} I_{n}(n-1 / 2) w\right)\right\} d t$

$-\int\left(\left(\partial_{t} Q_{M}\right) I_{n}(n-1 / 2) w, I_{n}(n-1 / 2) w\right) d t$

$+i \int\left(I_{n}(n-1 / 2)\left(D_{t}-i \theta\right) w,\left(Q_{M}-Q_{M}^{M}\right) I_{n}(n-1 / 2) w\right) d t$

$+2 \operatorname{Im} \int\left(\left[I_{n}(n-1 / 2), Q_{M}\right] w, I_{n}(n-1 / 2)\left(D_{t}-i \theta\right) w\right) d t$

$-\theta \int\left(I_{n}(n-1 / 2) w,\left(Q_{M}-Q_{M}^{*}\right) I_{n}(n-1 / 2) w\right) d t$,

where $Q_{M}$ is defined by (2.10).

From the same reasoning as in Section 3, the second term of the right-hand of (4.1) is equivalent to

$$
\begin{aligned}
& (n-1 / 2) \int\left\{\left(Q_{M} I_{n}(n-3 / 2) w, I_{n}(n-1 / 2) w\right)\right. \\
& \left.\quad+\left(Q_{M} I_{n}(n-1 / 2) w, I_{n}(n-3 / 2) w\right)\right\} d t, \bmod c(n)[u]_{-N}^{2}
\end{aligned}
$$

First we observe $I_{n}(n-1 / 2) * Q_{2} I_{n}(n-3 / 2)+I_{n}(n-3 / 2) * Q_{2} I_{n}(n-1 / 2)$. Since $Q_{2}(t, x, \xi) \in J^{2 p} S^{2}$, from Proposition 2.7, it follows that

$$
\begin{aligned}
& I_{n}(n-1 / 2)^{*} Q_{2} I_{n}(n-3 / 2)+I_{n}(n-3 / 2) * Q_{2} I_{n}(n-1 / 2) \\
& =2 I_{n}(n-1)^{*} Q_{2} I_{n}(n-1) \\
& \quad+I_{n}(n-1 / 2)^{*} J_{-}(p)^{*} A J_{-}(-1) I_{n}(n-1 / 2), \\
& \quad \text { with } A \in J^{p-2} S^{1}, \bmod S^{-\infty} .
\end{aligned}
$$

Here the inequality $p \geqq 2$ implies that $A \in J^{0} S^{1}$. Remarking that $J_{-}(p-1)^{*}\langle D\rangle J_{-}(p-1)$ $\in J^{2 p-2} S^{1}$, from the similar arguments as above we have

$$
\begin{aligned}
I_{n}( & n-1 / 2)^{*} J_{-}(p-1)^{*}\langle D\rangle J_{-}(p-1) I_{n}(n-3 / 2) \\
& +I_{n}(n-3 / 2)^{*} J_{-}(p-1)^{*}\langle D\rangle J_{-}(p-1) I_{n}(n-1 / 2) \\
= & 2 I_{n}(n-1)^{*} J_{-}(p-1)^{*}\langle D\rangle J_{-}(p-1) I_{n}(n-1) \\
& +I_{n}(n-1 / 2)^{*} J_{-}(p)^{*} A J_{-}(-1) I_{n}(n),
\end{aligned}
$$

with $A \in J^{p-2-1 / 2} S^{0}, \bmod S^{-\infty}$. Using (4.3) and (4.4), one can estimate (4.2) from below by 


$$
\begin{aligned}
& (2 n-1) \int\left(Q_{M} \mathbb{I}_{n}(n-1) w, \mathbb{I}_{n}(n-1) w\right) d t \\
& -c(n, M) \int\left\|\langle D\rangle \mathcal{J}_{-}(p) I_{n}(n-1 / 2) w\right\|^{2} d t \\
& -c(n, M) \int\left\|J_{-}(-1) \mathbb{I}_{n}(n-1 / 2) w\right\|^{2} d t \\
& -c(n, M) \int\left\|J_{-}(-1) I_{n}(n) w\right\|^{2} d t \\
& \bmod c(n, M)[u]_{-N}^{2} .
\end{aligned}
$$

Next consider $\mathbb{I}_{n}(n-1 / 2) *\left(Q_{M}^{*}-Q_{M}\right) I_{n}(n-1 / 2)=\mathbb{I}_{n}(n-1 / 2) *\left(Q_{2}^{*}\right.$ $\left.-Q_{2}\right) I_{n}(n-1 / 2)$. Writing $Q_{2}^{*}-Q_{2}=\tilde{Q}_{1}+\tilde{Q}_{0}$ with $\tilde{Q}_{1} \in J^{2 p-2} S^{1+4 n \sigma}, \tilde{Q}_{0} \in J^{0} S^{4 n \sigma}$, Proposition 2.7 gives that

$$
\begin{aligned}
\mathbb{I}_{n}(n-1 / 2) *\left(Q_{2}^{*}-Q_{2}\right) I_{n}(n-1 / 2) \\
\quad=\mathbb{I}_{n}(n-1 / 2)^{*} J_{-}(p)^{*} A J_{-}(-1) I_{n}(n)+\mathbb{I}_{n}(n)^{*} J_{-}(-1) * B J_{-}(-1) I_{n}(n) \\
\quad=I_{n}(n-1 / 2)^{*} J_{-}(p)^{*} \tilde{A} \mathbb{I}_{n}(n-1 / 2)+\mathbb{I}_{n}(n)^{*} J_{-}(-1)^{*} \tilde{B} \mathbb{I}_{n}(n-1 / 2),
\end{aligned}
$$

with $A \in J^{p-2+1 / 2} S^{1}, \tilde{A} \in J^{p-2} S^{1}, B \in J S^{0}, \tilde{B} \in J^{1 / 2} S^{0}, \bmod S^{-\infty}$.

From these, noting that $A, \tilde{A} \in S_{1-\sigma, \sigma}^{1}, B, \tilde{B} \in S_{1-\sigma, \sigma}^{0}$, it follows that

$$
\begin{aligned}
& \theta\left|\left(\mathbb{I}_{n}(n-1 / 2) w,\left(Q_{M}-Q_{M}\right) I_{n}(n-1 / 2) w\right)\right| \\
& \leqq c(n) \theta^{1 / 2}\left\|\langle D\rangle J_{-}(p) I_{n}(n-1 / 2) w\right\|^{2} \\
& +c(n) \theta^{3 / 2}\left\|J_{-}(-1) I_{n}(n) w\right\|^{2}, \bmod c(n) \theta^{1 / 2}|u|_{-N, \theta}^{2}, \\
& \left|\left(I_{n}(n-1 / 2)\left(D_{t}-i \theta\right) w,\left(Q_{M}-Q_{M}\right) I_{n}(n-1 / 2) w\right)\right| \\
& \leqq c(n)\left\|I_{n}(n-1 / 2)\left(D_{t}-i \theta\right) w\right\|^{2} \\
& \quad+c(n)\left\|\langle D\rangle J_{-}(p) I_{n}(n-1 / 2) w\right\|^{2} \\
& \quad+c(n)\left\|J_{-}(-1) I_{n}(n) w\right\|^{2}, \bmod c(n)|u|_{-N}^{2} .
\end{aligned}
$$

Where $|u|_{-N}^{2}=\|u\|_{-N}^{2}+\left\|\left(D_{t}-i \theta\right) u\right\|_{-N}^{2},|u|_{-N, \theta}^{2}=\theta^{\frac{1}{2}}\|u\|_{-N}^{2}+\theta^{-1 / 2}\left\|\left(D_{t}-i \theta\right) u\right\|_{-N}^{2}$.

Now estimate

$\left[I_{n}(n-1 / 2), Q_{M}\right]=\left[I_{n}(n-1 / 2), Q_{2}\right]+\mathbb{M}\left[I_{n}(n-1 / 2), J_{-}(p-1) *\langle D\rangle J_{-}(p-1)\right]$.

From Propositions 2.5 and 2.7 , taking into account that $p \geqq 2, Q_{2} \in J^{2 p} S^{2}$ and $J_{-}(p$ $-1)^{*}\langle D\rangle J_{-}(p-1) \in J^{2 p-2} S^{1}$, it is easy to see that $\left[I_{n}(n-1 / 2), Q_{M}\right]=A J_{-}(p) I_{n}(n-1 / 2)+B J_{-}(-1) I_{n}(n)$, with $A \in J^{0} S^{1}, \quad B \in$ $J^{1 / 2} S^{0}, \bmod S^{-\infty}$. Hence we have 
(4.8) $\left\|\left[I_{n}(n-1 / 2), Q_{M}\right] w\right\|^{2} \leqq c(n, M)\left\|\langle D\rangle J_{-}(p) I_{n}(n-1 / 2) w\right\|^{2}$ $+c(n, M)\left\|J_{-}(-1) I_{n}(n) w\right\|^{2}, \bmod c(n, M)|u|_{-N}^{2}$.

Finally we consider $I_{n}(n-1 / 2) *\left(\partial_{t} Q_{M}\right) I_{n}(n-1 / 2)$. We set

$$
A(t, x, \xi)=\partial_{t} Q_{2}(t, x, \xi) J_{-}(t, x, \xi)^{-2 p+1} \in J^{0} S^{2} .
$$

Remark that $A$ does not depend on $n$. Since $\partial_{t} Q_{2} \in J^{2 p-1} S^{2}$, Propositions 2.5 and 2.7 imply that

$$
\begin{aligned}
& I_{n}(n-1 / 2)^{*}\left(\partial_{t} Q_{2}\right) I_{n}(n-1 / 2)=I_{n}(n-1)^{*} J_{-}(p)^{*} A J_{-}(p) I_{n}(n-1) \\
& \quad+I_{n}(n-1 / 2)^{*} J_{-}(p)^{*} B J_{-}(p) I_{n}(n-1 / 2), B \in J^{-3} S^{1} \subset J^{0} S^{2}, \bmod S^{-\infty}
\end{aligned}
$$

Whereas taking into account that $\partial_{t}\left\{J_{-}(p-1)^{*}\langle D\rangle J_{-}(p-1)\right\} \in J^{2 p-3} S^{1}$, we have

$$
\begin{gathered}
I_{n}(n-1 / 2) * \partial_{t}\left\{J_{-}(p-1) *\langle D\rangle J_{-}(p-1)\right\} I_{n}(n-1 / 2) \\
\quad=I_{n}(n-1 / 2) * J_{-}(p) * \tilde{B} J_{-}(-1) I_{n}(n-1 / 2),
\end{gathered}
$$

with $\tilde{B} \in J^{p-2} S^{1} \subset J^{0} S^{1}, \bmod S^{-\infty}$. Thus the following inequality follows immediately from (4.9), (4.10) and Lemma 2.1,

$$
\begin{aligned}
& \left|\left(\left(\partial_{t} Q_{M}\right) I_{n}(n-1 / 2) w, I_{n}(n-1 / 2) w\right)\right| \leqq C_{1}^{2}\left\|\langle D\rangle J_{-}(p) I_{n}(n-1) w\right\|^{2} \\
& \quad+c(n, M)\left\|\langle D\rangle J_{-}(p) I_{n}(n-1 / 2) w\right\|^{2} \\
& \quad+c(n, \mathbb{M})\left\|J_{-}(-1) I_{n}(n-1 / 2) w\right\|^{2},
\end{aligned}
$$

$\bmod c(n, \mathbb{M})|u|_{-N}^{2}$, where $C_{1}=\sup \left|A(t, x, \xi)\langle\xi\rangle^{-2}\right|$.

Combining the inequalities (4.6), (4.7), (4.8) and (4.11), we get

Proposition 4.1.

$$
\begin{aligned}
& 2 \operatorname{Im} \int\left(I_{n}(n-1 / 2) Q_{M} w, I_{n}(n-1 / 2)\left(D_{t}-i \theta\right) w\right) d t \\
& \quad \geqq 2 \theta \operatorname{Re} \int\left(Q_{M} I_{n}(n-1 / 2) w, I_{n}(n-1 / 2) w\right) d t \\
& \quad+(2 n-1) \operatorname{Re} \int\left(Q_{M} I_{n}(n-1) w, I_{n}(n-1) w\right) d t \\
& \quad-C_{1}^{2} \int\left\|\langle D\rangle J_{-}(p) I_{n}(n-1) w\right\|^{2} d t-c(n) \int\left\|I_{n}(n-1 / 2)\left(D_{t}-i \theta\right) w\right\|^{2} d t \\
& \quad-c(n, M) \int\left\|J_{-}(-1) I_{n}(n-1 / 2) w\right\|^{2} d t \\
& \quad-c(n, M) \theta^{1 / 2} \int\left\|\langle D\rangle J_{-}(p) I_{n}(n-1 / 2) w\right\|^{2} d t
\end{aligned}
$$


$-c(n, M) \theta^{3 / 2} \int\left\|J_{-}(-1) I_{n}(n) w\right\|^{2} d t, \bmod c(n, M) \theta^{1 / 2}[u]_{-N, \theta}^{2}$.

Now take $L \geqq 2 n \sigma+N$ and fix $M$ so that Lemma 2.2 holds. Then Lemma 2.2 implies that

$$
\begin{aligned}
& (2 n-1) \operatorname{Re}\left(Q_{M} I_{n}(n-1) w, I_{n}(n-1) w\right)+2^{-1} n^{2}\left\|J_{-}(-1) I_{n}(n-1) w\right\|^{2} \\
& \quad \geqq c_{1} n\left\|\langle D\rangle J_{-}(p) I_{n}(n-1) w\right\|^{2}, c_{1}>0, \\
& 2 \theta \operatorname{Re}\left(Q_{M} I_{n}(n-1 / 2) w, I_{n}(n-1 / 2) w\right)+c_{2} n \theta\left\|J_{-}(-1) I_{n}(n-1 / 2) w\right\|^{2} \\
& \quad \geqq \tilde{c}_{1} \theta\left\|\langle D\rangle J_{-}(p) I_{n}(n-1 / 2) w\right\|^{2}, \tilde{c}_{1}>0 .
\end{aligned}
$$

Hence using these inequalities, Propositions 3.1 and 4.1 show that

\section{Lemma 4.1.}

$-2 \operatorname{Im} \int\left(I_{n}(n-1 / 2)\left[\left(D_{t}-i \theta\right)^{2}-Q_{M}\right] \alpha_{n}^{-} u, I_{n}(n-1 / 2)\left(D_{t}-i \theta\right) \alpha_{n}^{-} u\right) d t$

$$
\begin{aligned}
& \geqq c_{1} n \int\left\|\langle D\rangle J_{-}(p) I_{n}(n-1) \alpha_{n}^{-} u\right\|^{2} d t+c_{1} n \int\left\|I_{n}(n-1)\left(D_{t}-i \theta\right) \alpha_{n}^{-} u\right\|^{2} d t \\
& +c_{1} n^{2} \int\left\|J_{-}(-1) I_{n}(n-1) \alpha_{n}^{-} u\right\|^{2} d t+c_{2} \theta \int\left\|I_{n}(n-1 / 2)\left(D_{t}-i \theta\right) \alpha_{n}^{-} u\right\|^{2} d t \\
& +c_{2} \theta \int\left\|\langle D\rangle J_{-}(p) I_{n}(n-1 / 2) \alpha_{n}^{-} u\right\|^{2} d t+c_{2} n \theta \int\left\|J_{-}(-1) I_{n}(n-1 / 2) \alpha_{n}^{-} u\right\|^{2} d t \\
& +c_{2} \theta^{2} \int\left\|J_{-}(-1) I_{n}(n) \alpha_{n}^{-} u\right\|^{2} d t, \bmod c(n, N) \theta^{1 / 2}[u]_{-N, \theta}^{2},
\end{aligned}
$$

for $\theta \geqq \theta_{0}(n, N), n \geqq n\left(Q_{2}\right)$.

From the analogous arguments for $J_{+}$, we have,

\section{Lemma 4.2.}

$-2 \operatorname{Im} \int\left(J_{+}(-n-1 / 2)\left[\left(D_{t}-i \theta\right)^{2}-Q_{M}\right] \alpha_{n}^{+} u, J_{+}(-n-1 / 2)\left(D_{t}-i \theta\right) \alpha_{n}^{+} u\right) d t$ $\geqq c_{1} n \int\left\|\langle D\rangle J_{+}(p) J_{+}(-n-1) \alpha_{n}^{+} u\right\|^{2} d t+c_{1} n \int\left\|J_{+}(-n-1)\left(D_{t}-i \theta\right) \alpha_{n}^{+} u\right\|^{2} d t$ $+c_{1} n^{2} \int\left\|J_{+}(-1) J_{+}(-n-1) \alpha_{n}^{+} u\right\|^{2} d t+c_{2} \theta \int\left\|J_{+}(-n-1 / 2)\left(D_{t}-i \theta\right) \alpha_{n}^{+} u\right\|^{2} d t$ $+c_{2} \theta \int\left\|\langle D\rangle J_{+}(p) J_{+}(-n-1 / 2) \alpha_{n}^{+} u\right\|^{2} d t+c_{2} n \theta \int\left\|J_{+}(-1) J_{+}(-n-1 / 2) \alpha_{n}^{+} u\right\|^{2} d t$ $+c_{2} \theta^{2} \int\left\|J_{+}(-1) J_{+}(-n) \alpha_{n}^{+} u\right\|^{2} d t$, $\bmod c(n, N) \theta^{1 / 2}[u]_{-N, \theta}^{2}$, for $\theta \geqq \theta_{0}(n, N), n \geqq n\left(Q_{2}\right)$ 
If we note that

$$
\begin{aligned}
& I_{n}(n-1 / 2) * I_{n}(n-1 / 2) J(p-1) *\langle D\rangle J(p-1) \\
& \quad=I_{n}(n-1 / 2)^{*} J_{-}(p) * A^{-} I_{n}(n-1 / 2), \\
& J_{+}(-n-1 / 2)^{*} J_{+}(-n-1 / 2) J(p-1) *\langle D\rangle J(p-1) \\
& \quad=J_{+}(-n-1 / 2)^{*} J_{+}(p)^{*} A^{+} J_{+}(-n-1 / 2),
\end{aligned}
$$

with $A^{ \pm} \in J^{0} S^{1}$, mod $S^{-\infty}$, we can replace $Q_{M}$ by $Q_{2}$ in Lemmas 4.1 and 4.2 changing the constants $c_{2}, c(n, N)$ and $\theta_{0}(n, N)$.

Proposition 4.2. After having replaced $Q_{M}$ by $Q_{2}$, Lemmas 4.1 and 4.2 are also valid.

\section{§5. Estimates of the Communtators with $\alpha^{ \pm}$}

In this Section, we shall show that one can replace $\left[\left(D_{t}-i \theta\right)^{2}-Q_{2}\right] \alpha_{n}^{ \pm}$by $\alpha_{n}^{ \pm}\left[\left(D_{t}-i \theta\right)^{2}-Q_{2}\right]$ in Lemmas 4.1 and 4.2. Since $\alpha_{n}^{ \pm}\left(D_{t}-i \theta\right)^{2}=\left(D_{t}-i \theta\right)^{2} \alpha_{n}^{ \pm}$ $-2 D_{t} \alpha_{n}^{ \pm}\left(D_{t}-i \theta\right)-D_{t}^{2} \alpha_{n}^{ \pm}$, to do so we investigate $D_{t}^{2} \alpha_{n}^{ \pm}$and $D_{t} \alpha_{n}^{ \pm}$.

From Proposition 2.5, we see that

$$
\begin{gathered}
\sigma\left(I_{n}(n-1 / 2)^{*} I_{n}(n-1 / 2) D_{t}^{2} \alpha_{n}^{-}\right) \sim\langle\xi\rangle^{4 n \sigma} J_{-}(t, x, \xi)^{2 n-1} D_{t}^{2} \alpha_{n}^{-}+B, \\
\text { with } B \in J^{2 n-5} S^{4 n \sigma-1},
\end{gathered}
$$

then we define $A(t, x, \xi)$ by

$$
n J_{-}^{n-1} J_{+}^{-n-2}\langle\xi\rangle^{2 n \sigma} A(t, x, \xi) \alpha_{n}^{+}=\langle\xi\rangle^{4 n \sigma} J_{-}^{2 n-1} D_{t}^{2} \alpha_{n}^{-},
$$

that is

$$
\begin{gathered}
A(t, x, \xi)=-\left(\langle\xi\rangle^{2 \sigma} J_{+} J_{-}\right)^{n} \chi^{(2)}\left(-\sqrt{n}(t-\phi)\langle\xi\rangle^{\sigma}\right)\langle\xi\rangle^{2 \sigma} J_{+}^{2} \\
\left(D_{t}^{2} \alpha_{n}^{-}=-n \chi^{(2)}\left(-\sqrt{n}(t-\phi)\langle\xi\rangle^{\sigma}\right)\langle\xi\rangle^{2 \sigma}\right) .
\end{gathered}
$$

Since we have $J_{+}(t, x, \xi) J_{-}(t, x, \xi)=\langle\xi\rangle^{-2 \sigma}\left(1-(t-\phi(x, \xi))^{2}\langle\xi\rangle^{2 \sigma}\right)$ on $E_{1 / 4}$, it follows that

$$
\begin{aligned}
c_{1} \leqq & (1-1 / 4 n)^{ \pm n} \leqq\left(\langle\xi\rangle^{2 \sigma} J_{+}(t, x, \xi) J_{-}(t, x, \xi)\right)^{ \pm n} \\
& \leqq(1-1 / 16 n)^{ \pm n} \leqq c_{2} \quad \text { on } E_{1 / 4},
\end{aligned}
$$

with $c_{i}>0$ independent of $n$. 
Proposition 5.1. Let $a(t, x, \xi) \in J^{r} S^{m}$ and $\operatorname{supp}[a(t, x, \xi)] \subset E_{c}$ with some $c>0$. Then for any $\mu \in \mathbb{R}$, we have

$$
a(t, x, \xi) \in J^{r+\mu} S^{m+\sigma \mu} .
$$

Thus, using this proposition and (5.1), we have

$$
\begin{aligned}
& A(t, x, \xi) \in J^{0} S^{0} \\
& |A(t, x, \xi)| \leqq c \text { (independent of } n), \operatorname{supp}[A] \subset \operatorname{supp}\left[D_{t}^{2} \alpha_{n}^{-}\right] .
\end{aligned}
$$

From Propositions 2.5 and 5.1, it follows that

$$
\begin{gathered}
\sigma\left(I_{n}(n-1 / 2)^{*} I_{n}(n-1 / 2) D_{t}^{2} \alpha_{n}^{-}-n I_{n}(n-1)^{*} A J_{+}(-1) J_{+}(-n-1) \alpha_{n}^{+}\right) \sim b, \\
b \in J^{\mu} S^{-1+2 n \sigma+5 \sigma+\mu \sigma}, \quad \mu \in \mathbb{R}, \operatorname{supp}[b] \subset \operatorname{supp}\left[D_{t}^{2} \alpha_{n}^{-}\right] .
\end{gathered}
$$

Noting that $\alpha_{n}^{+}(t, x, \xi)=1$ on $\operatorname{supp}\left[D_{t}^{2} \alpha_{n}^{-}\right]$, Proposition 2.7 shows that

$$
\begin{aligned}
& I_{n}(n-1 / 2)^{*} I_{n}(n-1 / 2) D_{t}^{2} \alpha_{n}^{-}=n I_{n}(n-1)^{*} A J_{+}(-1) J_{+}(-n-1) \alpha_{n}^{+} \\
& \quad+I_{n}(n-1 / 2)^{*} B J_{+}(-1) J_{+}(-n-1 / 2) \alpha_{n}^{+},
\end{aligned}
$$

with $B \in J^{0} S^{-1+3 \sigma} \subset S_{1-\sigma, \sigma}^{0}, \bmod S^{-\infty}$, and hence

$$
\begin{aligned}
& \left|\left(I_{n}(n-1 / 2) D_{t}^{2} \alpha_{n}^{-} u, I_{n}(n-1 / 2)\left(D_{t}-i \theta\right) w\right)\right| \\
& \quad \leqq n^{3 / 2}\left\|A J_{+}(-1) J_{+}(-n-1) \alpha_{n}^{+} u\right\|^{2} \\
& \quad+n^{1 / 2}\left\|I_{n}(n-1)\left(D_{t}-i \theta\right) w\right\|^{2}+c(n)\left\|I_{n}(n-1 / 2)\left(D_{t}-i \theta\right) w\right\|^{2} \\
& \quad+c(n)\left\|J_{+}(-1) J_{+}(-n-1 / 2) \alpha_{n}^{+} u\right\|^{2}
\end{aligned}
$$

$\bmod c(n)|u|_{-N}^{2}$. Applying Lemma 2.1 to $A$, we get

$$
\begin{aligned}
& \left|\left(I_{n}(n-1 / 2) D_{t}^{2} \alpha_{n}^{-} u, I_{n}(n-1 / 2)\left(D_{t}-i \theta\right) \alpha_{n}^{-} u\right)\right| \\
& \leqq C^{2} n^{3 / 2}\left\|J_{+}(-1) J_{+}(-n-1) \alpha_{n}^{+} u\right\|^{2} \\
& \quad+n^{1 / 2}\left\|I_{n}(n-1)\left(D_{t}-i \theta\right) \alpha_{n}^{-} u\right\|^{2} \\
& \quad+c(n)\left\|I_{n}(n-1 / 2)\left(D_{t}-i \theta\right) \alpha_{n}^{-} u\right\|^{2} \\
& \quad+c(n)\left\|J_{+}(-1) J_{+}(-n-1 / 2) \alpha_{n}^{+} u\right\|^{2}, \bmod c(n)|u|_{-N}^{2}
\end{aligned}
$$

with $C$ independent of $n$.

From the same procedure, we can write

$$
\begin{aligned}
& I_{n}(n-1 / 2) * I_{n}(n-1 / 2) D_{t} \alpha_{n}^{-} \\
& \quad=n I_{n}(n-1) * A_{2} J_{+}(-n-1) \alpha_{n}^{+}+I_{n}(n-1 / 2) * B_{2} J_{+}(-n-1 / 2) \alpha_{n}^{+},
\end{aligned}
$$


with $B_{2} \in J^{0} S^{-1+3 \sigma} \subset S_{1-\sigma, \sigma}^{0}, \bmod S^{-\infty}$, where

$$
A_{2}(t, x, \xi)=i\left(\langle\xi\rangle^{2 \sigma} J_{+} J_{-}\right)^{n} \chi^{(1)}\left(-\sqrt{n}(t-\phi)\langle\xi\rangle^{\sigma}\right) J_{+}\langle\xi\rangle^{\sigma} .
$$

Since supp $\left[A_{2}\right] \cap \operatorname{supp}\left[D_{t} \alpha_{n}^{+}\right]=\phi$, it is clear that

$$
\begin{aligned}
& \mathbb{I}_{n}(n-1) * A_{2} J_{+}(-n-1) \alpha_{n}^{+}\left(D_{t}-i \theta\right) \\
& \quad=\mathbb{I}_{n}(n-1) * A_{2} J_{+}(-n-1)\left(D_{t}-i \theta\right) \alpha_{n}^{+}, \bmod S^{-\infty},
\end{aligned}
$$

and then we obtain

$$
\begin{aligned}
& \left|\left(\mathbb{I}_{n}(n-1 / 2) D_{t} \alpha_{n}^{-}\left(D_{t}-i \theta\right) u, I_{n}(n-1 / 2)\left(D_{t}-i \theta\right) \alpha_{n}^{-} u\right)\right| \\
& \leqq \sqrt{n}\left\|I_{n}(n-1)\left(D_{t}-i \theta\right) \alpha_{n}^{-} u\right\|^{2} \\
& \quad+C \sqrt{n}\left\|J_{+}(-n-1)\left(D_{t}-i \theta\right) \alpha_{n}^{+} u\right\|^{2} \\
& \quad+c(n)\left\|I_{n}(n-1 / 2)\left(D_{t}-i \theta\right) \alpha_{n}^{-} u\right\|^{2} \\
& \quad+c(n)\left\|J_{+}(-n-1 / 2)\left(D_{t}-i \theta\right) \alpha_{n}^{+} u\right\|^{2}, \bmod c(n)|u|_{-N}^{2}
\end{aligned}
$$

where $C$ does not depend on $n$.

Finally, we observe $\left[\alpha_{n}^{-}, Q_{2}\right]$. It is easily seen from Proposition 2.5 that

$$
\sigma\left(\left[\alpha_{n}^{-}, Q_{2}\right]\right) \sim a, a \in J^{2 p-2} S^{1}, \operatorname{supp}[a] \subset \mathbb{E}_{0}^{+} \cap \mathbb{E}_{1 / 2 \sqrt{n}}^{-} .
$$

Using Propositions 2.7 and 5.1, we have

$$
\begin{aligned}
& \mathbb{I}_{n}(n-1 / 2)^{*} \mathbb{I}_{n}(n-1 / 2)\left[\alpha_{n}^{-}, Q_{2}\right] \\
& \quad=\mathbb{I}_{n}(n-1 / 2) * A J_{+}(p) J_{+}(-n-1 / 2) \alpha_{n}^{+}+I_{n}(n-1 / 2) * B J_{+}(-1) J_{+}(-n) \alpha_{n}^{+},
\end{aligned}
$$

with $A \in J^{p-2} S^{1}, B \in J^{1 / 2} S^{0}, \bmod S^{-\infty}$. This shows that

$$
\begin{aligned}
& \left|\left(I_{n}(n-1 / 2)\left[\alpha_{n}^{-}, Q_{2}\right] u, I_{n}(n-1 / 2)\left(D_{t}-i \theta\right) \alpha_{n}^{-} u\right)\right| \\
& \leqq c(n)\left\|I_{n}(n-1 / 2)\left(D_{t}-i \theta\right) \alpha_{n}^{-} u\right\|^{2} \\
& \quad+c(n)\left\|\langle D\rangle J_{+}(p) J_{+}(-n-1 / 2) \alpha_{n}^{+} u\right\|^{2} \\
& \quad+c(n)\left\|J_{+}(-1) J_{+}(-n) \alpha_{n}^{+} u\right\|^{2}, \bmod c(n)|u|_{-N}^{2} .
\end{aligned}
$$

After making the same procedure for $J_{+}$, we get

\section{Lemma 5.1.}

$$
\begin{aligned}
& -2 \operatorname{Im} \int\left\{\left(\mathbb{I}_{n}(n-1 / 2) \alpha_{n}^{-}\left[\left(D_{t}-i \theta\right)^{2}-Q_{2}\right] u, I_{n}(n-1 / 2)\left(D_{t}-i \theta\right) \alpha_{n}^{-} u\right)\right. \\
& \left.\quad+\left(J_{+}(-n-1 / 2) \alpha_{n}^{+}\left[\left(D_{t}-i \theta\right)^{2}-Q_{2}\right] u, J_{+}(-n-1 / 2)\left(D_{t}-i \theta\right) \alpha_{n}^{+} u\right)\right\} d t \\
& \geqq \text { c } n \int\left\{\left\|I_{n}(n-1)\left(D_{t}-i \theta\right) \alpha_{n}^{-} u\right\|^{2}+\left\|J_{+}(-n-1)\left(D_{t}-i \theta\right) \alpha_{n}^{+} u\right\|^{2}\right\} d t
\end{aligned}
$$




$$
\begin{aligned}
& +\hat{c} n \int\left\{\left\|\langle D\rangle J_{-}(p) I_{n}(n-1) \alpha_{n}^{-} u\right\|^{2}+\left\|\langle D\rangle J_{+}(p) J_{+}(-n-1) \alpha_{n}^{+} u\right\|^{2}\right\} d t \\
& +c_{3} n^{2} \int\left\{\left\|J_{-}(-1) I_{n}(n-1) \alpha_{n}^{-} u\right\|^{2}+\left\|J_{+}(-1) J_{+}(-n-1) \alpha_{n}^{+} u\right\|^{2}\right\} d t \\
& +c_{4} \theta \int\left\{\left\|\langle D\rangle J_{-}(p) I_{n}(n-1 / 2) \alpha_{n}^{-} u\right\|^{2}+\left\|\langle D\rangle J_{+}(p) J_{+}(-n-1 / 2) \alpha_{n}^{+} u\right\|^{2}\right\} d t \\
& +c_{4} \theta \int\left\{\left\|I_{n}(n-1 / 2)\left(D_{t}-i \theta\right) \alpha_{n}^{-} u\right\|^{2}+\left\|J_{+}(-n-1 / 2)\left(D_{t}-i \theta\right) \alpha_{n}^{+} u\right\|^{2}\right\} d t \\
& +c_{4} n \theta \int\left\{\left\|J_{-}(-1) I_{n}(n-1 / 2) \alpha_{n}^{-} u\right\|^{2}+\left\|J_{+}(-1) J_{+}(-n-1 / 2) \alpha_{n}^{+} u\right\|^{2}\right\} d t \\
& +c_{4} \theta^{2} \int\left\{\left\|J_{-}(-1) I_{n}(n) \alpha_{n}^{-} u\right\|^{2}+\left\|J_{+}(-1) J_{+}(-n) \alpha_{n}^{+} u\right\|^{2}\right\} d t
\end{aligned}
$$

for $\theta \geqq \theta_{0}(n, N), n \geqq n\left(Q_{2}\right), \bmod c(n, N) \theta^{1 / 2}[u]_{-N, \theta}^{2}$.

\section{§6. Estimates of Lower Order Terms}

Let us consider $Q_{1}(t, x, \xi)=(t-\phi(x, \xi))^{p-1} q_{1}(t, x, \xi), \quad q_{1}(t, x, \xi) \in S_{1,0}^{1}$. Noting $Q_{1} \in J^{p-1} S^{1}$, we get

$$
\begin{aligned}
& I_{n}(n-1 / 2)^{*} I_{n}(n-1 / 2) Q_{1} \\
& \quad=I_{n}(n-1) * A J_{-}(p) I_{n}(n-1)+I_{n}(n-1 / 2)^{*} B J_{-}(p) I_{n}(n-1 / 2),
\end{aligned}
$$

with $A(t, x, \xi)=J_{-}(t, x, \xi)^{-p+1} Q_{1}(t, x, \xi) \in J^{0} S^{1}, B \in J^{-3} S^{0} \subset J^{0} S^{3 \sigma}, \bmod S^{-\infty}$. Here, we note that $A(t, x, \xi)$ does not depend on $n$. From the same reasoning which we have used in Section 4 , it follows that

$$
\begin{aligned}
& \left|\left(I_{n}(n-1 / 2) Q_{1} w, I_{n}(n-1 / 2)\left(D_{t}-i \theta\right) w\right)\right| \\
& \leqq \\
& \quad+4 \hat{c}^{-1} \hat{c} n\left\|I_{n}(n-1)\left(D_{t}-i \theta\right) w\right\|^{2} \\
& \quad+c(n)\left\|I_{n}(n-1 / 2)\left(D_{t}-i \theta\right) w\right\|^{2} \\
& \quad+c(n)\left\|\langle D\rangle J_{-}(p) I_{n}(n-1 / 2) w\right\|^{2}, \bmod c(n)|u|_{-N}^{2},
\end{aligned}
$$

with $C=\sup \left|J_{-}(t, x, \xi)^{-p+1} Q_{1}(t, x, \xi)\langle\xi\rangle^{-1}\right|$. On the other hand, Propositions 2.5, 2.7 and 5.1 show that

$$
\begin{aligned}
& I_{n}(n-1 / 2)^{*} I_{n}(n-1 / 2)\left[\alpha_{n}^{-}, Q_{1}\right] \\
& \quad=I_{n}(n-1 / 2)^{*} B J_{+}(-1) J_{+}(-n-1 / 2) \alpha_{n}^{+}, B \in J^{p-2} S^{0}, \bmod S^{-\infty} .
\end{aligned}
$$

Combining these, we get 


$$
\begin{aligned}
& \left|\left(I_{n}(n-1 / 2) \alpha_{n}^{-} Q_{1} u, I_{n}(n-1 / 2)\left(D_{t}-i \theta\right) \alpha_{n}^{-} u\right)\right| \\
& \leqq \\
& \quad+44^{-1} \hat{c} n\left\|I_{n}(n-1)\left(D_{t}-i \theta\right) \alpha_{n}^{-} u\right\|^{2} \\
& \quad+c(n)\left\|I_{n}(n-1 / 2)\left(D_{t}-i \theta\right) \alpha_{n}^{-} u\right\|^{2} \\
& \quad+c(n)\left\|\langle D\rangle J_{-}(p) I_{n}(n-1 / 2) \alpha_{n}^{-} u\right\|^{2} \\
& \quad+c(n)\left\|J_{+}(-1) J_{+}(-n-1 / 2) \alpha_{n}^{+} u\right\|^{2}, \bmod c(n)|u|_{-N}^{2}
\end{aligned}
$$

It is easy to see that for $R, Q_{0} \in S_{1,0}^{0}$,

$$
\begin{aligned}
& \left|\left(\mathbb{I}_{n}(n-1 / 2) \alpha_{n}^{-} Q_{0} u, I_{n}(n-1 / 2)\left(D_{t}-i \theta\right) \alpha_{n}^{-} u\right)\right| \\
& \leqq c(n)\left\|I_{n}(n-1 / 2)\left(D_{t}-i \theta\right) \alpha_{n}^{-} u\right\|^{2} \\
& +c(n)\left\|J_{-}(-1) I_{n}(n) \alpha_{n}^{-} u\right\|^{2} \\
& +c(n)\left\|J_{+}(-1) J_{+}(-n) \alpha_{n}^{+} u\right\|^{2}, \bmod c(n)|u|_{-N}^{2} \text {, } \\
& \left|\left(I_{n}(n-1 / 2) \alpha_{n}^{-} R\left(D_{t}-i \theta\right) u, I_{n}(n-1 / 2)\left(D_{t}-i \theta\right) \alpha_{n}^{-} u\right)\right| \\
& \leqq c(n)\left\|I_{n}(n-1 / 2)\left(D_{t}-i \theta\right) \alpha_{n}^{-} u\right\|^{2} \\
& +c(n)\left\|I_{n}(n-1 / 2) \alpha_{n}^{-}\left(D_{t}-i \theta\right) u\right\|^{2} \\
& +c(n)\left\|J_{+}(-n-1 / 2) \alpha_{n}^{+}\left(D_{t}-i \theta\right) u\right\|^{2}, \bmod c(n)|u|_{-N}^{2} \text {. }
\end{aligned}
$$

Here, we estimate $\left(D_{t}-i \theta\right) \alpha_{n}^{-} u$ in terms of $\alpha_{n}^{-}\left(D_{t}-i \theta\right) u$. Writing $I_{n}(n-1 / 2) D_{t} \alpha_{n}^{-}=\sqrt{n} A J_{+}(-1) J_{+}(-n-1 / 2) \alpha_{n}^{+}+B J_{+}(-1) J_{+}(-n) \alpha_{n}^{+}, \bmod S^{-\infty}$, with $A(t, x, \xi)=\left(J_{+} J_{-}\langle\xi\rangle^{2 \sigma}\right)^{n} J_{-}^{-1 / 2} J_{+}^{3 / 2}\langle\xi\rangle^{\sigma} \chi^{(1)}\left(-\sqrt{n}(t-\phi)\langle\xi\rangle^{\sigma}\right), B \in J^{-2-1 / 2} S^{-1}$, the same arguments as in Section 5 give that

$$
\begin{aligned}
& \left\|I_{n}(n-1 / 2) D_{t} \alpha_{n}^{-} u\right\|^{2} \\
& \quad \leqq C^{2} n\left\|J_{+}(-1) J_{+}(-n-1 / 2) \alpha_{n}^{+} u\right\|^{2}+c(n)\left\|J_{+}(-1) J_{+}(-n) \alpha_{n}^{+} u\right\|^{2},
\end{aligned}
$$

with $C=\sup |A(t, x, \xi)|$. Hence

$$
\begin{aligned}
& \left\|I_{n}(n-1 / 2)\left(D_{t}-i \theta\right) \alpha_{n}^{-} u\right\|^{2} \\
& \quad \geqq 2^{-1}\left\|I_{n}(n-1 / 2) \alpha_{n}^{-}\left(D_{t}-i \theta\right) u\right\|^{2}-C n\left\|J_{+}(-1) J_{+}(-n-1 / 2) \alpha_{n}^{+} u\right\|^{2} \\
& \quad-c(n)\left\|J_{+}(-1) J_{+}(-n) \alpha_{n}^{+} u\right\|^{2}, \bmod c(n)|u|_{-N}^{2},
\end{aligned}
$$

with $C$ independent of $n$. Using this inequality, one obtains

$$
\begin{aligned}
& n^{1 / 2}\left\|I_{n}(n-1)\left(D_{t}-i \theta\right) \alpha_{n}^{-} u\right\|^{2} \geqq 2^{-1} n^{1 / 2}\left\|I_{n}(n-1) \alpha_{n}^{-}\left(D_{t}-i \theta\right) u\right\|^{2} \\
& \quad-C n^{3 / 2}\left\|J_{+}(-1) J_{+}(-n-1) \alpha_{n}^{+} u\right\|^{2} \\
& \quad-c(n)\left\|J_{+}(-1) J_{+}(-n-1 / 2) \alpha_{n}^{+} u\right\|^{2}
\end{aligned}
$$




$$
\begin{aligned}
\theta^{1 / 2} \| & I_{n}(n-1 / 2)\left(D_{t}-i \theta\right) \alpha_{n}^{-} u\left\|^{2} \geqq 2^{-1} \theta^{1 / 2}\right\| I_{n}(n-1 / 2) \alpha_{n}^{-}\left(D_{t}-i \theta\right) u \|^{2} \\
& -C n \theta^{1 / 2}\left\|J_{+}(-1) J_{+}(-n-1 / 2) \alpha_{n}^{+} u\right\|^{2} \\
& -c(n) \theta^{1 / 2}\left\|J_{+}(-1) J_{+}(-n) \alpha_{n}^{+} u\right\|^{2},
\end{aligned}
$$

$\bmod c(n)|u|_{-N, \theta}^{2}$. On the other hand, the following inequalities are easily verified.

$$
\begin{aligned}
& n\left\|\langle D\rangle J_{-}(p) I_{n}(n-1) \alpha_{n}^{-} u\right\|^{2} \geqq 2^{-1} n\left\|\langle D\rangle^{2 n \sigma+1} J_{-}(n+p-1) \alpha_{n}^{-} u\right\|^{2} \\
& \quad-c(n)\left\|\langle D\rangle J_{-}(p) I_{n}(n-1 / 2) \alpha_{n}^{-} u\right\|^{2}, \\
& \theta\left\|\langle D\rangle J_{-}(p) I_{n}(n-1 / 2) \alpha_{n}^{-} u\right\|^{2} \geqq c_{0} \theta^{1 / 2}\left\|\langle D\rangle^{2 n \sigma+1} J_{-}(n+p-1 / 2) \alpha_{n}^{-} u\right\|^{2}, \\
& n^{2}\left\|J_{-}(-1) I_{n}(n-1) \alpha_{n}^{-} u\right\|^{2} \geqq 2^{-1} n^{2}\left\|\langle D\rangle^{2 n \sigma} J_{-}(n-2) \alpha_{n}^{-} u\right\|^{2} \\
& \quad-c(n)\left\|J_{-}(-1) I_{n}(n-1 / 2) \alpha_{n}^{-} u\right\|^{2}, \\
& n \theta\left\|J_{-}(-1) I_{n}(n-1 / 2) \alpha_{n}^{-} u\right\|^{2} \geqq 2^{-1} n \theta\left\|\langle D\rangle^{2 n \sigma} J_{-}(n-3 / 2) \alpha_{n}^{-} u\right\|^{2} \\
& \quad-c(n) \theta\left\|J_{-}(-1) I_{n}(n) \alpha_{n}^{-} u\right\|^{2}, \\
& \theta^{2}\left\|J_{-}(-1) I_{n}(n) \alpha_{n}^{-} u\right\|^{2} \\
& \quad \geqq c_{0} \theta^{3 / 2}\left\|\langle D\rangle^{2 n \sigma} J_{-}(n-1) \alpha_{n}^{-} u\right\|^{2}, \bmod c(n) \theta|u|_{-N, \theta}^{2},
\end{aligned}
$$

for $\theta \geqq \theta_{0}(n)$. The following is also immediate.

$$
I_{n}(n-1 / 2)^{*} I_{n}(n-1 / 2)=I_{n}(n-1) * B I_{n}(n), B \in J^{0} S^{0}, \bmod S^{-\infty} \text {. }
$$

Using the inequalities (6.2) through (6.7) and the corresponding inequalities for $J_{+}$, we can rewrite Lemma 5.1 as follows,

Lemma 6.1. Let $P_{\theta}=\left(D_{t}-i \theta\right)^{2}-\sum_{j=0}^{2} Q_{j}+R\left(D_{t}-i \theta\right)$, then

$$
\begin{aligned}
c(n, N) \int\left\|P_{\theta} u\right\|_{-2 N}^{2} d t+c(n, N) \int\left\|\mathbb{P}_{\theta} u\right\|_{n, 0,0}^{2} d t \\
\geqq \widehat{c}_{1} n^{1 / 2} \int\left\|\left(D_{t}-i \theta\right) u\right\|_{n, 0,1}^{2} d t+\widehat{c}_{2} n \int\|u\|_{n, 1,1-p}^{2} d t \\
\quad+\widehat{c}_{3} \theta^{1 / 2} \int\left\|\left(D_{t}-i \theta\right) u\right\|_{n, 0,1 / 2}^{2} d t+\widehat{c}_{3} \theta^{1 / 2} \int\|u\|_{n, 1,1 / 2-p}^{2} d t \\
\quad+\widehat{c}_{3} n^{2} \int\|u\|_{n, 0,2}^{2} d t+\widehat{c}_{3} n \theta \int\|u\|_{n, 0,3 / 2}^{2} d t+\widehat{c}_{3} \theta^{3 / 2} \int\|u\|_{n, 0,1}^{2} d t
\end{aligned}
$$

$\bmod c(n, N) \theta[u]_{-N, \theta}^{2}, n \geqq c\left(Q_{2}\right) C, \theta \geqq \theta_{0}(n, N)$, where $C=\sup \mid J(t, x$, $\xi)^{-p+1}\langle\xi\rangle^{-1} Q_{1}(t, x, \xi) \mid$. 
§7. Estimates of Error Terms amd Proolis of Theorems

Our aim in this section is to prove the following proposition, and complete the proof of theorems.

Proposition 7.1. We have

$$
\begin{aligned}
& \int\left\|P_{\theta} u\right\|_{-2 N}^{2} d t+\theta^{1 / 2} \int\left\|\left(D_{t}-i \theta\right) u\right\|_{n, 0,1 / 2}^{2} d t+\theta^{3 / 2} \int\|u\|_{n, 0,1}^{2} d t \\
& \quad \geqq c(n, N) \theta^{5 / 4} \int\left\|\left(D_{t}-i \theta\right) u\right\|_{-N}^{2} d t+c(n, N) \theta^{11 / 4} \int\|u\|_{-N}^{2} d t \\
& \quad \geqq c(n, N) \theta^{7 / 4}[u]_{-N, \theta}^{2},
\end{aligned}
$$

for $\theta \geqq \theta_{0}(n, N), N \geqq 1$, with $c(n, N)>0$.

Proof. First we note that

$$
-2 \operatorname{Im} \int\left(\left(D_{t}-i \theta\right)^{2} u,\left(D_{t}-i \theta\right) u\right) d t \geqq \theta \int\left\|\left(D_{t}-i \theta\right) u\right\|^{2} d t+\theta^{3} \int\|u\|^{2} d t .
$$

On the other hand, it is easily seen that

$$
\begin{gathered}
2 \operatorname{Im} \int\left(Q_{2} u,\left(D_{t}-i \theta\right) u\right) d t=2 \theta \operatorname{Re} \int\left(Q_{2} u, u\right) d t-\int\left(\left(\partial_{t} Q_{2}\right) u, u\right) d t \\
-i \int\left(\left(D_{t}-i \theta\right) u,\left(Q_{\overrightarrow{2}}^{*}-Q_{2}\right) u\right) d t+\theta \int\left(u_{9}\left(Q_{\overrightarrow{2}}^{*}-Q_{2}\right) u\right) d t \\
\geqq-c \int\left\|\left(D_{t}-i \theta\right) u\right\|^{2} d t-c \int\|\langle D\rangle u\|^{2} d t-c \theta \int\left\|\langle D\rangle^{1 / 2} u\right\|^{2} d t
\end{gathered}
$$

Remarking that

$\langle D\rangle^{-2 N} P_{\theta}=P_{\theta}\langle D\rangle^{-2 N}+q\langle D\rangle^{-2 N}+r\left(D_{t}-i \theta\right)\langle D\rangle^{-2 N}$, with $q \in S_{1,0}^{1}, r \in S_{1,0}^{-1}$,

we have

$$
\begin{gathered}
\int\left\|P_{\theta} u\right\|_{-2 N}^{2} d t \geqq c_{0} \theta^{2} \int\left\|\left(D_{t}-i \theta\right) u\right\|_{-2 N}^{2} d t+c_{0} \theta^{4} \int\|u\|_{-2 N}^{2} d t \\
-c \theta \int\|u\|_{-2 N+1}^{2} d t-c \theta^{2} \int\|u\|_{-2 N+1 / 2}^{2} d t, \text { for } \theta \geqq \theta_{0}(N) .
\end{gathered}
$$

Next, we observe \|\|$u \|_{n, 0,1}^{2}$ and $\left\|\left(D_{t}-i \theta\right) u\right\|_{n, 0,1 / 2}^{2}$. From Proposition 2.6, one can find $A \in J^{n+1} S^{0}$ so that

$$
\alpha_{4 n}^{+}=A J_{+}(-n-1) \alpha_{n}^{+}, \bmod S^{-\infty} .
$$


Since $\alpha_{n}^{+}(t, x, \xi)=1$ on $\operatorname{supp}\left[\alpha_{4 n}^{+}(t, x, \xi)\right]$, taking into account that $\operatorname{supp}\left[1-\alpha_{4 n}^{+}\right] \subset$ $\{t-\phi(x, \xi) \leqq 0\}$, Proposition 2.6 also gives that

$$
\begin{aligned}
& 1-\alpha_{4 n}^{+}=B\langle D\rangle^{2 n \sigma} J_{-}(n-1) \alpha_{n}^{-} \\
& \quad \text { with } B \in J^{-n+1} S^{-2 n \sigma} \subset J S^{-n \sigma}, \bmod S^{-\infty}(n \geqq 0) .
\end{aligned}
$$

Preceding two equalities imply that

$$
1=A J_{+}(-n-1) \alpha_{n}^{+}+B\langle D\rangle^{2 n \sigma} J_{-}(n-1) \alpha_{n}^{-}, \bmod S^{-\infty},
$$

and hence

$$
\begin{aligned}
& \|u\|^{2} \leqq c(n, N)\|u\|_{n, 0,1}^{2}+c(n, N)\|u\|_{-N}^{2}, \\
& \left\|\left(D_{t}-i \theta\right) u\right\|^{2} \leqq c(n, N)\left\|\left(D_{t}-i \theta\right) u\right\|_{n, 0,1 / 2}^{2}+c(n, N)\left\|\left(D_{t}-i \theta\right) u\right\|_{-N}^{2} .
\end{aligned}
$$

Whereas, the inequalities

$$
2 \theta^{11 / 4}\|u\|_{-N}^{2} \leqq \theta^{3 / 2}\|u\|^{2}+\theta^{4}\|u\|_{-2 N}^{2}, 2 \theta^{5 / 4}\|v\|_{-N}^{2} \leqq \theta^{1 / 2}\|v\|^{2}+\theta^{2}\|v\|_{-2 N}^{2},
$$

show that

$$
\begin{gathered}
c(n, N) \theta^{11 / 4}\|u\|_{-N}^{2} \leqq \theta^{3 / 2}\|u\|_{n, 0,1}^{2}+\theta^{4}\|u\|_{-2 N}^{2}, \\
c(n, N) \theta^{5 / 4}\left\|\left(D_{t}-i \theta\right) u\right\|_{-N}^{2} \leqq \theta^{1 / 2}\left\|\left(D_{t}-i \theta\right) u\right\|_{n, 0,1 / 2}^{2}+\theta^{2}\left\|\left(D_{t}-i \theta\right) u\right\|_{-2 N}^{2},
\end{gathered}
$$

for $\theta \geqq \theta_{0}(n, N)$. In view of $-N \geqq-2 N+1(N \geqq 1),(7.1)$ and (7.3) prove this proposition.

Now, by virtue of Proposition 7.1, the following lemma follows immediately from Lemma 6.1 .

Lemma 7.1. For $n \geqq c\left(Q_{2}\right) C, N \geqq 1, \theta \geqq \theta_{0}(n, N)$, we have

$$
\begin{aligned}
c(n, N) & \int\left\|P_{\theta} u\right\|_{-2 N}^{2} d t+c(n, N) \int\left\|P_{\theta} u\right\|_{n, 0,0}^{2} d t \\
\geqq & \widehat{c}_{1} n^{1 / 2} \int\left\|\left(D_{t}-i \theta\right) u\right\|_{n, 0,1}^{2} d t+\widehat{c}_{2} n \int\|u\|_{n, 1,1-p}^{2} d t \\
& +\widehat{c}_{3} \theta^{1 / 2} \int\left\|\left(D_{t}-i \theta\right) u\right\|_{n, 0,1 / 2}^{2} d t+\widehat{c}_{3} \theta^{1 / 2} \int\|u\|_{n, 1,1 / 2-p}^{2} d t \\
& +c_{4} \theta^{5 / 4} \int\left\|\left(D_{t}-i \theta\right) u\right\|_{-N}^{2} d t+c_{4} \theta^{11 / 4} \int\|u\|_{-N}^{2} d t,
\end{aligned}
$$

where $C=\sup \left|J(t, x, \xi)^{-p+1}\langle\xi\rangle^{-1} Q_{1}(t, x, \xi)\right|, c_{4}=c_{4}(n, N)$ and $c\left(Q_{2}\right)$ depends 
only on $Q_{2}$.

We shall deduce Theorems 1.2 and 1.3 from Lemma 7.1. To show Theorem 1.2 , it suffices to note that $\left(D_{t}-i \theta\right) e^{-\theta t} u=e^{-\theta t} D_{t} u$.

Next we shall prove Theorem 1.3. We operate $\langle D\rangle^{s+1}$ to $P$, then

$$
\begin{aligned}
& \langle D\rangle^{s+1} P=\tilde{P}\langle D\rangle^{s+1}, \tilde{P}=D_{t}^{2}-Q_{2}+\left(Q_{1}+\tilde{Q}_{1}\right)+\tilde{Q}_{0}+\tilde{R} D_{t}, \\
& \tilde{Q}_{0} \in S_{1,0}^{0}, \tilde{R} \in S_{1,0}^{0},
\end{aligned}
$$

where $\tilde{Q}_{1}=\left[\langle D\rangle^{s+1}, Q_{2}\right]\langle D\rangle^{-s-1} \in J^{2 p-2} S^{1}$. Thus the term $\tilde{Q}_{1}$ is the only one which must be considered. From Proposition 2.7, we get

$$
\begin{aligned}
& I_{n}(n-1 / 2)^{*} I_{n}(n-1 / 2) \tilde{Q}_{1}=I_{n}(n-1 / 2)^{*} B J_{-}(p) I_{n}(n-1 / 2), \\
& B \in J^{p-2} S^{1}, \bmod S^{-\infty},
\end{aligned}
$$

and then

$$
\begin{aligned}
& \left|\left(I_{n}(n-1 / 2) \tilde{Q}_{1} w, I_{n}(n-1 / 2)\left(D_{t}-i \theta\right) w\right)\right| \leqq c(n)\left\|I_{n}(n-1 / 2)\left(D_{t}-i \theta\right) w\right\|^{2} \\
& \quad+c(n)\left\|\langle D\rangle J_{-}(p) I_{n}(n-1 / 2) w\right\|^{2}, \bmod c(n)|u|_{-N}^{2}
\end{aligned}
$$

Therefore in Lemma 7.1, we can replace $P_{\theta}$ by $\tilde{P}_{\theta}$ without any change. On the other hand, since $\langle D\rangle^{2 n \sigma} J_{-}(n) \in J^{n} S^{2 n \sigma} \subset S_{1-\sigma, \sigma}^{2 n \sigma}, J_{+}(-n) \in J^{-n} S^{0} \subset S_{1-\sigma, \sigma}^{n \sigma}$, it follows that $\left\|\langle D\rangle^{s+1} v\right\|_{n, 0,0}^{2} \leqq c(n)\|v\|_{2 n \sigma+s+1}^{2}$, and hence

$$
\begin{aligned}
& \left\|\tilde{P}_{\theta}\langle D\rangle^{s+1} u\right\|_{n, 0,0}^{2} \leqq c(n)\left\|P_{\theta} u\right\|_{2 n \sigma+s+1}^{2}, \\
& \left\|\tilde{P}_{\theta}\langle D\rangle^{s+1} u\right\|_{-2 N}^{2}=\left\|P_{\theta} u\right\|_{-2 N+s+1}^{2} .
\end{aligned}
$$

Then, replacing $P_{\theta}, u$ by $\tilde{P}_{\theta},\langle D\rangle^{s+1} u$ and taking $N=1$ in Lemma 7.1, we have Theorem 1.3.

\section{Refieremees}

[1] R. Beals and C. Fefferman, Spatially inhomogeneous pseudo-differential operators, I, Comm. Pure Appl. Math., 27, (1974), 1-24.

[2] L. Boutet de Monvel, Hypoelliptic operators with double characteristics and related pseudodifferential operators, Comm. Pure Appl. Math., 27, (1974), 585-639.

[3 ] D. Gourdin, Problème de Cauchy faiblement hyperbolique, Bull. Sc. Math., 105, (1982), 259272.

[4] V. Ja. Ivrii and V. M. Petkov, Necessary conditions for the Cauchy problem for nonstrictly hyperbolic equations to be well-posed, Russian Math. Surveys, 29, (1974), 1-70. 
[5] V. Ja. Ivrii, Sufficient conditions for regular and completely regular hyperbolicity, Trans. Moscow Math. Soc., 1, (1978), 1-65.

[6] N. Iwasaki, Cauchy problems for effectively hyperbolic equations (a special case), preprint (1983).

[7] H. Kumanogo, Pseudo Differential Operators, M. I. T. Press (1982).

[8] A. Menikoff, The Cauchy problem for weakly hyperbolic equations, Amer. Jour. Math., 97, (1975), 548-558.

[9] T. Nishitani, Some remarks on the Cauchy problem for weakly hyperbolic equations, Jour. Math. Kyoto Univ., 17, (1977), 245-258.

[10] - Sur les opérateurs fortement hyperboliques qui ne dépendent que du temps, Séminaire sur les équations aux dérivees partielles hyperboliques et holomorphes, Univ. de Paris VI, (1981-82).

[11] , On the Cauchy problem for weakly hyperbolic equations, Comm. P. D. E., 3, (1978), 319-333.

[12] The Cauchy problem for weakly hyperbolic equations of second order, Comm. $P$. D. E., 5, (1980), 1273-1296.

[13] - A necessary and sufficient condition of hyperbolicity for second order equations, (to appear in Jour. Math. Kyoto Univ.).

[14] O. A. Oleinik, On the Cauchy problem for weakly hyperbolic equations, Comm. Pure Appl. Math., 23, (1970), 569-586.

[15] V. M. Petkov, Le problème de Cauchy pour une classe d'équations non fortement hyperboliques à caracteristiques doubles (en russe), J. bulgare de Mathématiques. 1, (1975), 372-380. 